\title{
Fotografien und Industrie - Historischer Überblick
}

Wann die Industrialisierung im Zarenreich begann, lässt sich nicht eindeutig beantworten und hängt vom jeweiligen Verständnis des Begriffs Industrialisierung ab. Sowjetische Historiker datierten die ersten Anfänge der Industrialisierung im Zarenreich auf das 17. Jahrhundert. ${ }^{1}$ Für sie war ausschlaggebend, dass in dieser Zeit adelige Unternehmer damit begannen, Kapital anzuhäufen und erste kleinerer Unternehmen zu gründen. Allerdings handelte es sich bei diesen frühen Formen von Industrie mit Blick auf das gesamte Zarenreich um Ausnahmeerscheinungen, und die Betriebe besaßen kaum Maschinen, so dass die Produktion auf Handarbeit basierte. ${ }^{2}$ Gemeinhin werden in der Forschung eher die Regierungszeit Peter I. (1672-1725) und dessen Reformen als Beginn gewerblicher Großproduktion in Russland angesehen. Der Zar förderte unter anderem die Herstellung von Salz und Pottasche und setzte sich besonders für die Entstehung von metallverarbeitendem Gewerbe im Ural ein, wo er erste Unternehmen gründete. ${ }^{3}$ Diese Betriebe erfüllen jedoch ebenfalls nicht die Voraussetzung, um von Industrialisierung zu

* In dieser Darstellung wird die Entwicklung bis zu Beginn des Ersten Weltkriegs skizziert. Die Situation während des Kriegs wird im entsprechenden Kapitel näher behandelt. Siehe hierzu: Kapitel „Die Fabrik wird salonfähig - der Erste Weltkrieg“, S. 325-368.

1 Beispielsweise: Pavel Alekseevič Chromov: Očerki èkonomiki feodalizma v Rossii, Moskva 1957, S. 109-134; Pavel Alekseevič Chromov: Ėkonomičeskoe razvitie Rossii. Očerki èkonomiki Rossii s drevnajšich vremen do Velikoj Oktjabr'skoj revoljucii, Moskva 1967, S. 278; M. D. Kurmačev: Vopros o pervonačal'nom nakoplenii v Rossii v sovetskoj istoričeskoj literature, in: L. G. Beskrovnyj; E. I. Zaozerskaja; A. A. Preobraženskij (Hrsg.): K voprosu o pervonačal'nom nakoplenii v Rossii (XVII-XVIII vv.), Moskva 1958, S. 9-37; F. Ja. Poljanskij: Pervonačal'noe nakoplenie kapitala v Rossii, Moskva 1958. Zu diesen Debatten siehe auch: Nikolaj I. Pavlenko: Historiographische Bemerkungen zur Genese des Kapitalismus in Rußland, in: Peter Hoffmann; Heinz Lemke (Hrsg.): Genesis und Entwicklung des Kapitalismus in Rußland. Studien und Beiträge, Berlin 1973, S. 1-25, S. 1-13.

2 Blackwell: The Beginnings of Russian Industrialization, S. 13-14.

3 Roger Portal: L'Oural au XVIII e siècle. Étude d'histoire économique et sociale, Paris 1950, S. 25-51; Roger Portal: The Industrialization of Russia, in: H. Habakkuk, M. Postman (Hrsg.): The Cambridge Economic History of Europe, Bd. 6. The Industrial Revolutions and after: Incomes, Population and Technological Change, Cambridge 1965, S. 801-872, S. 8o6; Blackwell: The Beginnings of Russian Industrialization, S. 17; Vincent Barnett: The Revolutionary Russian Economy, 1890-1914. Ideas, Debates and Alternatives, London 2004, S. $6-7$. 
sprechen: Sie stützten sich auf Heimarbeit oder proto-industrielle Strukturen und nutzten in der Produktion selten Maschinen. ${ }^{4}$

Nach dieser ersten Initiative stärkte besonders Zarin Katharina II. (17291796) die Entwicklung des zweiten Wirtschaftssektors, indem sie nichtadeligen Kaufleuten das Recht zubilligte, Unternehmen zu gründen, und Betrieben erlaubte, Leibeigene zu besitzen. ${ }^{5}$ Dies ermöglichte Unternehmern, eine ausgebildete Arbeiterschaft heranzuziehen. ${ }^{6}$ Insgesamt zeigten diese ersten Anreize von staatlicher Seite jedoch nur eine begrenzte Wirkung. Einerseits war der europäische Markt besonders an den Rohstoffen aus dem Zarenreich interessiert, gefertigte Waren aus Russland stießen hingegen auf eine geringe Nachfrage. Andererseits war der Kreditmarkt in Russland nur schwach entwickelt, was Neugründungen und Investitionen generell erschwerte. ${ }^{7}$ Besonders adelige Landbesitzer hatten wenig Interesse, dies zu ändern, weil sie um ihre führende soziale Stellung fürchteten. ${ }^{8}$ Ihnen kam die protektionistische Politik des Finanzministeriums zugute, die ihnen durch hohe Einfuhrzölle und Einfuhrverbote ausländischer Waren ihre wirtschaftliche Monopolstellung sicherte. ${ }^{9}$ In der Folge entwickelte sich die russische Industrie nur langsam und blieb auf einem wenig modernisierten Stand. Die

4 Zur Definition von Industrialisierung: Dietrich Hilger; Lucian Hölscher: Industrie, Gewerbe, in: Otto Brunner; Werner Conze; Reinhart Koselleck (Hrsg.): Geschichtliche Grundbegriffe. Historisches Lexikon zur politischen Sprache in Deutschland, Bd. 3, Stuttgart 1982, S. 237304, S. $282-283$.

5 Heller: Geschichte des modernen Unternehmertums, S. 7; Dietrich Geyer: „Gesellschaft“ als staatliche Veranstaltung. Bemerkungen zur Sozialgeschichte der russischen Staatsverwaltung im 18. Jahrhundert, in: Jahrbücher für Geschichte Osteuropas, Jg. 14/1966, Heft 1, S. 21-50, S. 27.

6 Pavel Alekseevič Chromov: Očerki èkonomiki dokapitalističeskoj Rossii, Moskva 1988, S. 144145; Darlay: Factory, S. 51. Zur Förderung der Industrie im 18. Jahrhundert siehe auch: Pavel Alekseevič Chromov: Ėkonomičeskoe razvitie Rossii v XIX-XX vekach. 1800-1917, Moskva 1950, S. 38-45.

7 Blackwell: The Beginnings of Russian Industrialization, S. 88-89.

8 Zum Verhältnis von russischen Adeligen und Kaufleuten: Arcadius Kahan: Die Kosten der „Verwestlichung“ in Rußland. Adel und Ökonomie im 18. Jahrhundert, in: Dietrich Geyer (Hrsg.): Wirtschaft und Gesellschaft im vorrevolutionären Rußland, Köln 1975, S. 53-82, S. 68-71. Zahlreiche russische Adelige gerieten im Laufe des 19. Jahrhunderts in finanzielle Schwierigkeiten. Siehe hierzu: Andreas Grenzer: Adel und Landbesitz im ausgehenden Zarenreich. Der russische Landadel zwischen Selbstbehauptung und Anpassung nach Aufhebung der Leibeigenschaft, Göttingen 1995, insbesondere S. 100-108.

9 Portal: The Industrialization of Russia, S. 803, 805. Die protektionistische Politik setzte sich auch deshalb durch, weil ihre Vertreter führende Positionen in der russischen Regierung innehatten. Die Befürworter des Freihandels besetzten eher untere Ränge und waren dadurch in einer schwächeren Position. Blackwell: The Beginnings of Russian Industrialization, S. 124-125. 
Einfuhrzölle wirkten als Garant für den Absatz der Produktion, erhöhten jedoch die Kosten für importierte maschinelle Ausstattung: Eine Spindel in einer russischen Baumwollfabrik war in der Anschaffung dreimal teurer als in England..$^{10}$ Erst in den $1840 e r$ und 185 oer Jahren kam es im Bereich der Textilindustrie zu einer starken Mechanisierung der Fabriken;"11 es kann von einem ersten Industrialisierungsschub gesprochen werden. ${ }^{12}$ Die Dampfmaschinen waren englische Importe, so dass die noch schwach ausgebaute metallverarbeitende Industrie im Zarenreich kaum von diesen Investitionen profitierte. ${ }^{13}$ Es war besonders die Leichtindustrie, die in den 1840er und 1850er Jahren die russische Industrie dominierte.

\subsection{Wachsende Bedeutung von Industrie und Fotografie im ausgehenden Zarenreich}

Etwa in diese Zeit fällt auch die offizielle Entdeckung oder Erfindung der Fotografie im Jahr $1839 .{ }^{14}$ Nur wenige Tage, nachdem der Franzosen Louis Daguerre $(1787-1851)$ das Verfahren der Daguerreotypie in Paris vorgestellt hatte, setzten Redakteure russischer Zeitungen sich schon damit auseinander, wofür die neue Technik verwendet werden könne. ${ }^{15}$ Hier zeigt sich, wie schnell die Zirkulation von Informationen erfolgte und dass die Eliten des Zarenreichs weit vernetzt und über Entwicklungen in Westeuropa gut unterrichtet waren. Die erste fotografische Aufnahme in Russland entstand wenige Monate später:

10 Zu den Zollabgaben mussten die Fabrikanten außerdem für hohe Transportkosten sowie für das Salär der Spezialisten aufkommen, die die Maschinen montierten. Gestwa, ProtoIndustrialisierung, S. 160; Kahan: Wirtschafts- und Sozialgeschichte Rußlands, S. 38.

11 In England setzte dieser Prozess bereits Mitte des 18. Jahrhunderts ein, nachdem der Engländer John Kay (1704-1780) das erste mechanisch bewegte Weberschiffchen entwickelt hatte. Wolfgang Ebert: Kathedralen der Arbeit. Historische Industriearchitektur in Deutschland, Tübingen, Berlin 1996, S. 9.

12 Blackwell: The Beginnings of Russian Industrialization, S. 42-46, 50.

13 Portal: The Industrialization of Russia, S. 810.

14 Bereits vor dem offiziellen Datum, an dem das Verfahren in Paris präsentiert wurde, fanden in unterschiedlichen Ländern Experimente mit ähnlichen Verfahren statt. Popov: Iz istorii rossijskoj fotografii, S. 8-9. Zur Frage, ob es sich bei der Fotografie um eine Erfindung oder um eine Entdeckung handelte, siehe: Peter Geimer: Bilder aus Versehen. Eine Geschichte fotografischer Erscheinungen, Hamburg 2010, S. 49-56.

15 Außerdem schickte die Akademie der Wissenschaften in St. Petersburg einen Abgesandten mit dem Auftrag nach Paris und London, mehr über die neue Technik herauszufinden. Elena Barchatova: The First Photographs in Russia, in: David Elliot (Hrsg.): Photography in Russia 1840-1940, London 1992, S. 24-30, S. 26; Elliot: The Photograph in Russia, S. 11-12. 
Sie zeigt die im Bau befindliche Isaakskathedrale in St. Petersburg. ${ }^{16}$ Bereits im folgenden Jahr hatten erste fotografische Ateliers eröffnet, in denen, wer es sich leisten konnte, eine Daguerreotypie von sich anfertigen ließ. ${ }^{17}$ Die Betreiber benötigten ein großzügiges Startkapital, denn allein eine Kamera kostete 1839 rund 550 Rubel. ${ }^{18}$

Ende der 1840er Jahre begann nach der Hochzeit der Daguerreotypie eine neue Ära in der (russischen) Fotografie. Das von William Henry Fox Talbot entwickelte Prinzip des Positiv-Negativ-Verfahren setzte sich im Zarenreich durch. Jetzt ließen sich, anders als bei der Daguerreotypie, von einem aufgenommenen Negativ mehrere Abzüge anfertigen. ${ }^{19}$ Dies förderte die Attraktivität der neuen Bilder, erhöhte die Einnahmequellen der Fotografen und steigerte die Erfolgsaussichten in diesem Berufsfeld. Während die Entwicklung der russischen Wirtschaft aufgrund der Niederlage des Zarenreichs im Krimkrieg (1853-1856) stagnierte und in eine Krise geriet, entwickelte sich die Fotografie schnell zu einem florierenden Gewerbe. In allen Regionen des Imperiums ergriffen Menschen den Beruf des Fotografen. Zunächst waren dies besonders häufig Ausländer, bald wandten sich aber auch Bürger des Russischen Reichs dem neuen Gewerbe zu. ${ }^{20}$ Doch diese Trennung zwischen russischen und ausländischen Lichtbildnern existierte so klar nicht. Von Beginn an waren russische Fotografen auch international vernetzt. Sie absolvierten häufig Teile ihrer Ausbildung in Westeuropa und präsentierten ihre Abzüge auf internationalen Ausstellungen. ${ }^{21}$ Bereits 1849 gewann beispielsweise Sergej L'vovič Levickij (1819-1898) als erster russischer Fotograf für seine Landschaftsaufnahmen eine Goldmedaille auf der Pariser Gewerbeausstellung. ${ }^{22}$

Das fotografische Gewerbe im Zarenreich zeigte sich von der Wirtschaftskrise im Zuge des Krimkriegs unbeeindruckt und profitierte stattdessen von neuen technischen Entwicklungen. $\mathrm{Zu}$ nennen ist hier besonders die französische Entwicklung der Carte de visite. ${ }^{23}$ Ähnlich wie in Deutschland

16 Barchatova: Wissenschaft? Handwerk? Kunst!, S. 8.

17 Barchatova: The First Photographs in Russia, S. 26-27; Tat'jana Nikolaevna Šipova: Fotografy Moskvy, 1839-1930. Biografičeskij slovar'-spravočnik, Moskva 20o6, S. 6-7. Eine Daguerreotypie ist eine fotografische Aufnahme auf einer Metallplatte. Diese Fotografien waren Unikate, gaben den aufgezeichneten Gegenstand seitenverkehrt wieder und zeigten je nach Lichteinfall oder Bearbeitungstechnik eine Negativ- oder Positivaufnahme. Dies berichtete die Zeitung Moskovskie Vedomosti (Moskauer Anzeiger). Šipova: Fotografy Moskvy (2006), S. 5-6.

19 Šipova: Fotografy Moskvy (2006), S. 12-13.

20 Elliot: The Photograph in Russia, S. 12.

21 Barchatova: Wissenschaft? Handwerk? Kunst!, S. 18-20, 24-25.

22 Barchatova: Wissenschaft? Handwerk? Kunst!, S. 9.

23 Jens Jäger: Fotografie und Geschichte, Frankfurt a. M., New York 2009, S. 62. 
und Großbritannien war in Russland die Portraitfotografie das am weitesten verbreitete Genre. ${ }^{24}$ Die kleinformatigen Aufnahmen der Carte de visite hatten im Unterschied zu klassischen Portraits den Vorteil, dass sie vergleichsweise günstig waren, was sie sehr populär machte. ${ }^{25}$ Es entwickelte sich mit dem Visitformat erstmals ein standardisiertes Format, das es erlaubte, bei der Entwicklung der Bilder Maschinen einzusetzen. ${ }^{26}$ Vornehmlich Mitglieder der russischen Mittelschicht und des Adels tauschten und sammelten die Abbildungen. Einem Zeitgenossen nach waren 1858 Visitenkartenportraits so beliebt, dass die Anzahl der fotografischen Einrichtungen im Zarenreich stark zunahm. ${ }^{27}$ Dieser Entwicklung war zuträglich, dass staatliche Behörden zunächst verhältnismäßig offen gegenüber der Eröffnung von Ateliers waren und es administrativ wenige Hindernisse gab. ${ }^{28}$

Die russische Niederlage im Krimkrieg hatte das Zarenreich an den Rand eines Staatsbankrotts gebracht. Außerdem war offensichtlich geworden, dass Russland den Anschluss an die industrielle Entwicklung Westeuropas verpasst hatte. Nachdem erste Schritte zur Industrialisierung der Wirtschaft bereits vor $185^{\circ}$ auf den Weg gebracht worden waren, beschleunigte sich der Prozess in den Nachkriegsjahren. Zar Alexander II. (1818-1881) stieß mit den Großen Reformen eine Vielzahl an gesellschaftlichen wie wirtschaftlichen Veränderungen an, um die Ursachen der beschämenden Niederlage zu beseitigen. ${ }^{29}$ Ein großer gesellschaftlicher Einschnitt war 1861 die Abschaffung der Leibeigenschaft. Auf die russische Industrie hatte diese Maßnahme jedoch nur begrenzte Auswirkungen. Fabriken, die auf die Arbeit von Leibeigenen angewiesen waren, gerieten unmittelbar nach 1861 zunächst in eine Krise, beispielsweise im Ural, wo Metallarbeiter zum Teil „kopflos“ ihren Arbeitsplatz aufgaben. ${ }^{30}$ Allerdings war es den Menschen auch nach der Aufhebung der Leibeigenschaft nicht erlaubt, frei ihren Wohnsitz und Arbeitsplatz zu wählen. Darum hatte letztlich die Abschaffung der Kopfsteuer in den Jahren 1882-1885 einen größeren Einfluss auf die Industrie, weil sie die Bewegungsfreiheit der Bauern erheblich erweiterte. ${ }^{31}$

\footnotetext{
24 Barchatova: Russkaja svetopis', S. 234; Jäger: Gesellschaft und Photographie, S. 144.

25 Tat'jana Saburova: Early Masters of Russian Photography, in: David Elliot (Hrsg.): Photography in Russia 1840-1940, London 1992, S. 31-40, S. 35.

26 Starl: Im Prisma des Fortschritts, S. 15.

27 Saburova: Early Masters of Russian Photography, S. 35.

28 Popov: Iz istorii rossijskoj fotografii. S. 18.

29 Heiko Haumann: Geschichte Russlands, München 1996, S. 351-365.

30 Michail Ivanovič Tugan-Baranovsky: Geschichte der russischen Fabrik, Berlin 1900, S. $371-372$.

31 Portal: The Industrialization of Russia, S. 811.
} 
Insgesamt verzeichnete die russische Industrie in den 186oer Jahren einen ersten Boom. Maßgeblich hierfür war die Gründung der russischen Staatsbank (Gosudarstvennyj bank Rossijskoj Imperii) 1860, die den größten Teil des Finanz- und Kreditwesens kontrollierte. Sie diente als Vorbild für weitere Gründungen privater Handelsbanken, die als Aktiengesellschaften organisiert waren. ${ }^{32}$ Außerdem finanzierte die russische Staatsbank den Bau der Eisenbahnen. Diese Projekte lockten in großem Stil auch ausländische Investoren $\mathrm{an}^{33}$ und markierten nach der ersten Mechanisierung in den 1840er und 1850er Jahren eine zweite Phase der Industrialisierung im Zarenreich. Neben den Aufträgen für die metallverarbeitende Industrie wirkten sich insbesondere die durch den Ausbau der Infrastruktur rückläufigen Transportkosten positiv auf die russische Wirtschaft aus. ${ }^{34}$ Infolge dessen erwirtschafteten die Betriebe Anfang der 1870er Jahre hohe Gewinne, die die Fabrikanten in neue technische Ausstattung und in den Ausbau neuer Produktionsstätten reinvestierten. ${ }^{35}$ Nach den Großen Reformen lösten Kaufleute den Adel langsam als Modernisierungsagenten ab. ${ }^{36}$

Die wirtschaftliche und gesellschaftliche Aufbruchsstimmung im Zarenreich bildete den Hintergrund, vor dem in den 186oer Jahren auch die Popularität der Fotografien weiter anstieg. Sie entwickelten sich zu einem festen Bestandteil im Alltag der russischen Bevölkerung. ${ }^{37}$ Abzüge standen auf Schreibtischen, Kaminsimsen und Regalen der wohlhabenden Schichten der russischen Gesellschaft, teilweise schmückten ganze ,Ikonostasen' aus Fotografien die Wohnräume. ${ }^{38}$ Nicht nur für die sozial besser gestellten Schichten, die sich ein eigenes Portrait leisten konnten, waren die Fotoateliers von Bedeutung. Die Ateliers entwickelten sich dank ihrer Schaufenster auch für weniger

32 Olga Crisp: Studies in the Russian Economy before 1914, London 1976, S. 121-123; Hildermeier: Geschichte Russlands, S. 1153; Portal: The Industrialization of Russia, S. 813. Hildermeier: Geschichte Russlands, S. 1141. Zur Bedeutung ausländischer Investoren und ausländischen Wissens siehe auch: McKay: Pioneers for Profit, S. 12-37.

34 Die hohen Kosten für russische Waren, sowohl aus der industriellen Produktion als auch aus der Landwirtschaft, waren in erster Linie auf die hohen Transportkosten zurückzuführen. Blackwell: The Beginnings of Russian Industrialization, S. 8o; Schenk: Russlands Aufbruch in die Moderne?, S. 191.

35 William Blackwell: The Industrialization of Russia. A Historical Perspective, Arlington Heights ${ }^{3} 1994$, S. 42; Portal: The Industrialization of Russia, S. 820-822.

36 Lindner: Unternehmer und Stadt in der Ukraine, S. 463.

37 Galina A. Miroljubova; Tat'jana A. Petrova: Das Goldene Zeitalter der Photographie. Die photographische Sammlung der Ermitage, Leningrad, in: Nikolaj Nikolaevič Rachmanov (Hrsg.): Das Russland der Zaren. Photographien von 1839 bis zur Oktoberrevolution, Berlin 1989, S. 100-121, S. 102.

38 Miroljubova; Petrova: Das Goldene Zeitalter der Photographie, S. 109. 
wohlhabende Menschen zu wichtigen Institutionen. Der englische Journalist George Augustus Sala (1828-1895) beschrieb in seinen Aufzeichnungen, die er während eines Russlandaufenthalts im Jahr 1856 anfertigte, die Auslage des Ladens für Druckerzeugnisse und Fotografie der Familie Daziaro in der Hauptstadt St. Petersburg. Laut Sala ließen sich die aktuellen Ereignisse besser und schneller an den Fotografien in den Ladenvitrinen ablesen als in einer Tageszeitung. Stürbe eine wichtige Persönlichkeit, so könne am selben oder spätestens am nächsten Tag ihr Portrait bei Daziaro erworben werden. ${ }^{39}$ Dieses Beispiel verdeutlicht, wie wichtig Laufkundschaft und die Lage eines Fotoateliers waren. Um möglichst viele Kunden zu erreichten, bevorzugten Lichtbildner für ihre Einrichtungen Standorte an belebten Straßen oder in der Nähe von Klöstern oder Kathedralen, die zahreiche Menschen anzogen. ${ }^{40}$

Auch in kleineren Städten nahmen immer mehr Fotoateliers ihren Betrieb auf, um die auch in der Provinz größer werdende Nachfrage an Bildern zu bedienen. Für die Eröffnung eines Ateliers musste ab 1862 in Moskau der Generalgouverneur, in St. Petersburg der Stadthauptmann und in anderen Städten der jeweilige regionale Gouverneur seine Erlaubnis erteilen. ${ }^{41}$ Neben der Zustimmung, auf der vermerkt war, wo sich die Einrichtung befinden solle, benötigte der Antragsteller zwei vertrauenswürdige Bürgschaften. Außerdem musste er eine jährliche Gebühr von etwa 20 Rubel entrichten ${ }^{42}$ und dafür sorgen, dass der Feuerschutz sowie eine gute Belüftung gesichert war und kein Wasser von den verwendeten Chemikalien verunreinigt wurde. ${ }^{43}$ Die Zensurbehörden verpflichteten die Lichtbildner, von allen Aufnahmen ihres Ateliers Abzüge anzufertigen. Diese mussten sie mit Inventarnummern versehen und in speziellen Alben aufbewahren, so dass jederzeit alle Bilder für die Behörden leicht zugänglich waren. ${ }^{44}$ Keine Fotografie durfte das Atelier verlassen, ohne

39 George Augustus Sala: A Journey due North. Being Notes of a Residence in Russia in the Summer of 1856, London 1859, S. 93-94.

40 Šipova: Fotografy Moskvy (2006), S. 11.

41 Popov: Iz istorii rossijskoj fotografii, S. 18; sowie die Korrespondenz aus Tula: GATO f. 9o, op. 1 t 47 , d. 41460.

42 Popov: Iz istorii rossijskoj fotografii, S. 19.

43 Tat'jana Nikolaevna Šipova:„DerZukunft zu Erinnerung“, Das Archiv der Literatur, Moskau, in: Nikolaj Nikolaevič Rachmanov (Hrsg.): Das Russland der Zaren. Photographien von 1839 bis zur Oktoberrevolution, Berlin 1989, S. 146-160, S. 147.

44 Popov: Iz istorii rossijskoj fotografii, S. 21. Allerdings konnte trotz intensiver Recherchen keines dieser Inventaralben für eine Analyse im Rahmen der vorliegenden Arbeit ausfindig gemacht werden. Es ist darum fraglich, ob das entsprechende Gesetz tatsächlich zur Anwendung kam oder ob die Zeitgenossen die Bestimmung in der Praxis tatsächlich umsetzten. Insgesamt besteht zu Fragen und konkreten Umsetzung der Bildzensur und zur Zensur von Fotografien im Zarenreich noch großer Forschungsbedarf. 
dass auf ihr der Name der Firma vermerkt gewesen wäre. ${ }^{45}$ Darüber hinaus regulierten Fotografieverbote die Wahl der Motive. Teilweise war der Zugang zu bestimmten Orten den Fotografen verboten, in anderen Fällen durften von bestimmten Ereignissen wie Militärparaden oder dem Stapellauf eines Schiffs nicht ohne explizite Genehmigung Fotografien angefertigt werden. ${ }^{46}$

Neben den professionellen Lichtbildnern nahm auch die Zahl der Amateure und Liebhaber (fotograf-ljubiteli) der neuen Technik zu. Beide Gruppen begannen in den 1870er Jahren, sich zusammenzuschließen und in Gesellschaften zu organisieren. Zunächst gründete die Kaiserlich Russländische Technische Gesellschaft (Imperatorskoe Rossijskoe Techničeskoe Obščestvo) 1866 eine Unterabteilung, die sich der Fotografie widmete. ${ }^{47}$ Die Gründung einer ersten selbstständigen fotografischen Gesellschaft, der Obščestvo rasprostranenie techničeskich znanij (Gesellschaft zu Verbreitung technischen Wissens), erfolgte 1871 und bald folgten weitere. ${ }^{48}$ Sowohl die Gesellschaften als auch Privatpersonen gaben eine große Zahl an fotografischen Zeitschriften heraus, die sich an professionelle Fotografen wie an Amateure richteten. ${ }^{49}$ Die Publikationen informierten ihre Leser über technische Neuentwicklungen, Ausstellungen oder Diskussionen zu bestimmten Stilrichtungen in der Fotografie. Teilweise handelte es sich um Texte russischer Autoren, in anderen Fällen veröffentlichten die Redaktionen Übersetzungen von Artikeln aus westeuropäischen Fachzeitschriften. Damit spiegeln die Periodika, wie international vernetzt russische Akteure im Bereich der Fotografie waren.

In der russischen Wirtschaft waren in den 186oer und 1870er Jahren Beziehungen zu internationalen Partnern ebenfalls sehr wichtig: Ausländische Kapitalimporte finanzierten zunächst große Teile des russischen Eisenbahnbaus. ${ }^{50}$ Nutznießer dieser umfangreichen Bauprojekte war die metallver-

45 Polnoe sobranie zakonov Rossijskoj imperii, Tom 40, 1865, čast' 1, zakon No. 41990 (06.04.1865), S. 397-406.

46 Barchatova: Russkaja svetopis', S. 49; Aleksandra Golovina: Für die Nachkommen aufbewahren. Die Photographische Sammlung des Zentralarchivs für Film- und Photodokumente, Leningrad, in: Nikolaj Nikolaevič Rachmanov (Hrsg.): Das Russland der Zaren. Photographien von 1839 bis zur Oktoberrevolution, Berlin 1989, S. 170-186, S. 172.

47 Barchatova: Wissenschaft? Handwerk? Kunst!, S. 12.

48 Popov: Iz istorii rossijskoj fotografii, S. 29-32; Barchatova: Wissenschaft? Handwerk? Kunst!, S. 20, 29-32.

49 Wobei die ersten Zeitschriften erschienen, bevor sich die ersten russischen Fotografen zu Gesellschaften zusammenschlossen. Barchatova: Wissenschaft? Handwerk? Kunst!, S. 9-19, 30. Zur russischen fotografischen Gesellschaft in Moskau: Tat'jana Saburova: Russkoe fotografičeskoe obščestvo v Moskve. 1894-1930, Moskva 2013.

$5^{\circ}$ Dietrich Geyer: Der russische Imperialismus. Studien über den Zusammenhang von innerer und auswärtiger Politik 1860-1914, Göttingen 1977, S. 105. 
arbeitende Industrie, die im Zarenreich an Bedeutung gewann. ${ }^{51}$ Ein weiterer Grund für das Wachstum dieses Industriezweigs war die Aufhebung der hohen Zölle auf die Importe von Roheisen. ${ }^{52}$ Mitte der 187oer Jahre wurden aus Kreisen russischer Unternehmer Stimmen laut, die von der Regierung eine protektionistischere Politik forderten, damit die russischen Hersteller sich neben der westeuropäischen Konkurrenz behaupten konnten. Diese Bitte griff Finanzminister Michail Christoforovič Rejtern (1820-1890) auf, und die russische Regierung untersagte 1876 die zollfreie Einfuhr von Schienen. Die Eisenbahngesellschaften erhielten die Auflage, mindestens die Hälfte ihres Bedarfs an Schienen aus der russischen Produktion zu decken. ${ }^{53}$ Noch bis Anfang der 189oer Jahre waren Schutzzölle eines der zentralen Mittel der Finanzpolitik des Zarenreichs. ${ }^{54}$

Die russische Regierung verfolgte neben dem Schutz der nationalen Wirtschaft die Strategie, russische Exporte zu steigern. Der Bau der Eisenbahn hatte die dafür nötige Infrastruktur geschaffen. Der größte Posten aller ins Ausland verkauften Güter war Getreide. ${ }^{55}$ Diese Exporte und die niedrigen

$5^{1} \quad$ Pavel G. Ryndzjunskij: Einige Probleme der sozioökonomischen Entwicklung Rußlands in der zweiten Hälfte des 19. Jahrhunderts, in: Peter Hoffmann; Heinz Lemke (Hrsg.): Genesis und Entwicklung des Kapitalismus in Rußland. Studien und Beiträge, Berlin 1973, S. 241-262, S. 261-262.

52 Hildermeier: Geschichte Russlands, S. 1142-1143.

53 Valerij I. Bovkin: Probleme der industriellen Entwicklung Rußlands, in: Dietrich Geyer (Hrsg.): Wirtschaft und Gesellschaft im vorrevolutionären Rußland, Köln 1975, S. 188-209, S. 191-192; Portal: The Industrialization of Russia, S. 816-818. Diese Strategie war dahingehend erfolgreich, dass in den 189oer Jahren der größte Teil der verlegten Eisenbahnschienen aus russischer Produktion stammte, allerdings teilweise aus Unternehmen mit ausländischem Kapital oder aus Betrieben, die unter ausländischer Ägide errichtet worden waren.

54 Die russische Regierung hoffte, mithilfe der erhobenen Zölle unter anderem die Kosten des Russisch-Osmanischen Kriegs (1877-1878) finanzieren zu können. Hildermeier: Geschichte Russlands, S. 1143. Zur russischen Schutzzollpolitik siehe auch: Arcadius Kahan: Russian Economic History. The Nineteenth Century, Chicago 1989, S. 19; Valentin Wittschewsky: Russlands Handels-, Zoll- und Industriepolitik von Peter dem Grossen bis auf die Gegenwart, Berlin 1905, S. 234; Dietrich Beyrau; Manfred Hildermeier: Von der Leibeigenschaft zur frühindustriellen Gesellschaft (1856-189o), in: Gottfried Schramm (Hrsg.): Handbuch der Geschichte Rußlands, Bd. 3/1. Von den autokratischen Reformen bis zum Sowjetstaat (1856-1945), Stuttgart 1983, S. 5-202, S. 116-118; Iosif F. Gindin: Rol' železnodorožnogo stroitel'stva v industrializacii Rossii i železnodorožnaja politika S. Ju. Vitte, in: S. Ju. Vitte: Sobranie sočinenij i dokumental'nych materialov v pjati tomach, tom. 1. Puti soobščenija i èkonomičeskoe razvitie Rossii, kniga 2, čast' 2, Moskva 2006, S. 583-622, S. 606.

55 Barry K. Goodwin; Thomas J. Grennes: Tsarist Russia and the World Wheat Market, in: Explorations in Economic History, Jg. 35/1998, Heft 4, S. 405-430. In der Forschung wurde immer wieder diskutiert, ob es sich bei diesen Exporten um „Hungerexporte“ 
europäischen Zinsen sorgten dafür, dass die Wirtschaft des Zarenreichs ab 1888 für ein Jahrzehnt schwarze Zahlen schrieb. ${ }^{56}$ Allerdings führte der massenhafte Export von Getreide 1891-1892 nach einer Missernte in vielen Gebieten des Russischen Reichs zu einer großen Hungersnot mit darauf folgenden Cholera- und Typhusepidemien. ${ }^{57}$ Die Regierung musste große Summen aufwenden, um die Bevölkerung zu versorgen. Die Situation verdeutlichte die wirtschaftliche Schwäche und das fragile soziale Gleichgewicht des Zarenreichs. Als Nikolaus II. 1894 den Thron bestieg, konnte er wirtschaftliche Reformen daher nicht verwehren. ${ }^{58}$ Treibende Kraft war hier der ebenfalls neu angetretene Finanzminister Sergej Jul'evič Vitte (1849-1915). ${ }^{59}$ Er wollte Russland schnellstmöglich industrialisieren, um dessen Unabhängigkeit zu stärken und zu verhindern, dass das Zarenreich zur Kolonie westeuropäischer Wirtschaftsmächte würde. ${ }^{60}$ Vitte wandte sich von der Schutzzollpolitik ab und setzte sich stattdessen dafür ein, die russische Wirtschaft für ausländische Investoren attraktiv zu machen und neue Märkte für Waren aus dem Zarenreich

gehandelt habe. Zu diesem Thema siehe beispielsweise: Boris Ananich: The Russian Economy and Banking System, in: Dominic Lieven (Hrsg.): The Cambridge History of Russia, Bd. 2. Imperial Russia, 1689-1917, Cambridge 2006, S. 394-425; Blackwell: The Industrialization of Russia, S. 28-29; Geyer: Der russische Imperialismus, S. 106; Portal: The Industrialization of Russia, S. 815 .

56 Hildermeier: Geschichte Russlands, S. 1144.

57 David Moon: The Environmental History of the Russian Steppes. Vasilii Dokuchaev and the Harvest Failure of 1891, in: Transactions of the Royal Historical Society, Jg. 15/2005, S. 149-174; Richard G. Robbins: Famine in Russia. 1891-1892. The Imperial Government Responds to a Crisis, New York 1975; Alfred Eisfeld; Guido Hausmann; Dietmar Neutatz (Hrsg.): Hungersnöte in Russland und in der Sowjetunion 1891-1947. Regionale, ethnische und konfessionelle Aspekte, Essen 2017; zu den Folgen, die die Hungersnot für das Ansehen und den Rückhalt der russischen Regierung in der Bevölkerung hatte siehe: Orlando Figes: Die Tragödie eines Volkes. Die Epoche der russischen Revolution 1891 bis 1924, Berlin 1998, S. 173-178.

$5^{8}$ Ananich: The Russian Economy and Banking System, S. 411.

59 L. I. Abalkin: Ėkonomičeskie vozzrenija i gosudarstvennaja dejatel'nost' S. Ju. Vitte, in: ders. u. a. (Hrsg.): Sobranie sočinenij i dokumental'nych materialov. Bd. 1. Puti soobščenija i èkonomičeskoe razvitie Rossii, Moskva 2002, S. 12-42.

6o Neutatz: Träume und Alpträume, S. 50-51; Thomas C. Owen: Industrialization and Capitalism, in:AbbottGleason (Hrsg.): A Companion to Russian History, Malden, Mass., Oxford 2009, S. 210-224, S. 217-218; Stefan Plaggenborg:Staatsfinanzen und Industrialisierung in Rußland 1881-1903. Die Bilanz der Steuerpolitik für Fiskus, Bevölkerung und Wirtschaft, Wiesbaden 1990; Heinz-Dietrich Löwe: Von der Industrialisierung zur ersten Revolution, 1890-1904, in: Gottfried Schramm (Hrsg.): Handbuch der Geschichte Rußlands, Bd. 3/1. Von den autokratischen Reformen zum Sowjetstaat (1856-1945), Stuttgart 1983, S. 203335 , S. $213-215$. 
zu erschließen. ${ }^{61}$ Eine wichtige Maßnahme hierfür war der Goldstandard für den russischen Rubel, den der Minister 1897 durchsetzte. ${ }^{62}$ Die Golddeckung der Währung sollte das Vertrauen der Investoren stärken und russische Anleihen international attraktiv machen. ${ }^{63}$ Für die Stahlindustrie gelang dies, um die Jahrhundertwende machte ausländisches Kapital hier circa 78 Prozent aller Investitionen aus. Weitere Impulse für die heimische Wirtschaft erhoffte sich Vitte von einem gigantischen Infrastrukturprojekt - dem Bau der Transsibirischen Eisenbahn. ${ }^{64}$ Die neue Linie erschloss einerseits potentielle Absatzmärkte, andererseits sicherte das Bauvorhaben Aufträge für russische Unternehmen. Die Funktionseliten verbanden mit dem Projekt die Hoffnung, dass die Transsibirische Eisenbahn Russland zum zentralen Umschlagplatz für den lukrativen Handel zwischen Ostasien und Westeuropa machen würde. ${ }^{65}$ Vitte bemühte sich neben der direkten Förderung der Montanindustrie und des Maschinenbaus darum, ${ }^{66}$ dass die positive Wirtschaftslage und die ausländischen Importe auch auf die Leichtindustrie ausstrahlten. Abgesehen von der Ausstattung mit neuen Maschinen setzten die Ausländer neue Produktionsverfahren und Organisationsformen in ihren Betrieben um,

61 Diese Periode kann als dritte Phase der Industrialisierung bezeichnet werden. In dieser Zeit etablierten sich in den Metropolen neue Industriezweige wie die Chemie- und Elektroindustrie.

62 Olga Crisp: Russian Financial Policy and the Gold Standard at the End of the Nineteenth Century, in: The Economic History Review, Jg. 6/1953, Heft 2, S. 156-172.

63 Hierzu beispielsweise: Gatrell: Reconceptualizing Russia's Industrial Revolution, S. 231; Robin Winks; Joan Neuberger: Europe and the Making of Modernity, 1815-1914, New York, Oxford 2005, S. 235-236; Francis Wcislo: Tales of Imperial Russia. The Life and Times of Sergej Witte, 1849-1915, Oxford 2011, S. 154-155, 159-161. Diese Reform ermöglichte maßgeblich, die sibirischen Goldvorkommen zu fördern. Portal: The Industrialization of Russia, S. 825. Zu Maßnahmen Vittes, die ausländische Investoren nach Russland locken sollten, siehe auch: Heller: Geschichte des modernen Unternehmertums, S. 16.

64 Als Vitte 1894 das Amt des Finanzministers übernahm, war dieses Projekt zwar bereits beschlossen, dennoch ist seine Person sehr eng mit der Durchführung der Baumaßnahmen verbunden. Hierzu: Steven Marks: Road to Power. The Trans-Siberian Railway and the Colonization of Asian Russia, 1850-1917, Ithaca 1991, S. 117-169; Wcislo: Tales of Imperial Russia, S. 154-156, 176-180.

65 Frithjof Benjamin Schenk: Das Zarenreich als Transitraum zwischen Europa und Asien. Russische Visionen und westliche Perzeptionen um die Jahrhundertwende, in: Martin Aust (Hrsg.): Globalisierung imperial und sozialistisch. Russland und die Sowjetunion in der Globalgeschichte 1851-1991, Frankfurt a. M., New York 2013, S. 41-63.

66 Allerdings kam diese Förderung nicht allen Gebieten gleichermaßen zugute. Während Unternehmen im Donbass viele Aufträge erhielten, profitierten Betriebe im Ural weniger von den Maßnahmen. Jurij Alekseevič Buranov: Akcionirovanie gorozavodskoj promyšlennosti Urala. 1861-1917 gg., Moskva 1982, S. 9. 
die russische Unternehmer teilweise übernahmen. ${ }^{67}$ Die Politik des Finanzministers war insgesamt sehr erfolgreich, und in der Wirtschaftsgeschichte gelten die 189oer Jahre als die Zeit des industriellen Take-off im Zarenreich. ${ }^{68}$ Allerdings muss betont werden, dass dieses Wachstum nicht allein auf einer kurzfristigen, staatlichen Initiative beruhte, sondern einen längerfristigen Prozess darstellte. ${ }^{69}$

In der Periode dieses starken Wirtschaftswachstums entwickelte sich im Zarenreich die Fotografie zu einem eigenständigen Wirtschaftszweig. In den 1860er Jahren hatte die Fotografie weder als Kunst, noch als Industrie gegolten, sondern war dem Handwerk zugerechnet worden. Dies galt nicht nur für das Zarenreich, auch auf der Weltausstellung 1862 in London hatten Lichtbildner ihre Ausstellungsplätze noch Seite an Seite mit Handwerksbetrieben. ${ }^{70} \mathrm{Ab}$ den 188oer Jahren fanden zunehmend mehr Menschen ihr finanzielles Auskommen im Wirtschaftsbereich der Fotografie, auch wenn die Zahlen für das Zarenreich verglichen mit Frankreich und Großbritannien relativ bescheiden ausfielen. 1882 gab es in Paris bereits 400 fotografische Einrichtungen, die 23.00o Personen ernährten. In London waren es zum gleichen Zeitpunkt 96o Ateliers, die für das Auskommen von 35.00o Menschen sorgten. In der russischen Hauptstadt hingegen arbeiteten in 104 Einrichtungen 800 bis 900 Personen. Für das gesamte Zarenreich sicherten die Erwerbsmöglichkeiten in 800 fotografische Einrichtungen die Existenzgrundlage von rund 40.000 Menschen. ${ }^{71}$ Darunter war auch die Fotografin Anna Petrovna Vjatkina, ${ }^{72}$ die ein Atelier in Kazan unterhielt, in dem neben ihr sechs Personen arbeiteten. Das Studio machte Ende der 1880er Jahre in einem Zeitraum von zwölf Monaten ca. 8.00o Rubel Umsatz, wobei Vjatkina 7.10o Rubel für Material und

67 Hildermeier: Geschichte Russlands, S. 1141, 1146.

68 Zum Konzept des industriellen Take-off: Walt Whitman Rostow: The World Economy. History and Prospect, London 1978.

69 Neutatz: Träume und Alpträume, S. 77; Winks; Neuberger: Europe and the Making of Modernity, S. 234-235; Nicholas V. Riasanovsky: Russian Identities. A Historical Survey, Oxford 2005, S. 188-189; Portal: The Industrialization of Russia, S. 836.

70 Šipova: „Der Zukunft zu Erinnerung“, S. 147; Stigneev: Vek fotografii, S. 19.

71 Hierzu werden jedoch auch die Familien der Angestellten gezählt. Barchatova: Russkaja svetopis', S. 219.

72 Die genauen Lebensdaten von Vjatkina sind leider nicht bekannt. Sicher ist jedoch, dass sie ab den 186oer Jahren ein Atelier betrieb und bis um die Jahrhundertwende Fotografien erschienen, die auf Karton aufgeklebt waren, der mit ihrem Namen bedruckt war. A. M. Osipov: Katalog Kazanskoj naučno-promyšlennoj vystavki. Sosotijaščej pod pokrovitel'stvom ego Imperatorskago Vysočestva Gosudarja naslednika Cesareviča. Knižno-tipografskij i fotografičeskij otdel, Kazan' 189o, S. 7 . 
sonstige Kosten ausgeben musste. ${ }^{73}$ Die 900 Rubel Gewinn zeigen, dass es sich um ein lukratives Geschäft handelte.

Die Menschen, die den Beruf des Fotografen ergreifen wollten, stammten aus unterschiedlichen sozialen Schichten. Im Gouvernement Tula wandte sich 1873 eine Hebamme mit der Bitte an den Gouverneur, in ihrem Haus ein Atelier einrichten zu dürfen. ${ }^{74}$ Weiter suchten Adelige, Kaufleute, Kleinbürger, Bauern, Ärzte, Studenten oder Postillions ihr Glück in dem neuen Beruf; die größte Gruppe der professionellen Fotografen waren Künstler. ${ }^{75}$ In der Forschungsliteratur zur Geschichte der russischen Fotografie im 19. Jahrhundert sind die untersuchten Protagonisten mehrheitlich männlich; es ergriffen allerdings auch zahlreiche Frauen den Beruf. ${ }^{76}$ Möglicherweise war es für sie ein Vorteil, dass sich das Berufsfeld des Fotografen erst neue etablierte, wodurch insgesamt neue Aufstiegschancen entstanden. ${ }^{77}$ Noch gab es kaum ausgebildete Organisationsformen und Hierarchien, die Frauen von der Ausübung des Berufs fernhielten. Ein ähnliches Phänomen lässt sich in der ebenfalls neu entstehenden Werbebranche beobachten: Frauen leiteten 1896 fünf von 13 Werbeagenturen in Moskau. ${ }^{78}$ Es ist zu vermuten, dass diese soziale Durchlässigkeit in gleicher Weise für Menschen aus weniger angesehenen Gesellschaftsschichten galt. Solange sie das nötige Kapital für die Eröffnung und den Unterhalt eines Fotostudios aufbringen konnten, stand ihnen der Zugang zu diesem Beruf offen. ${ }^{79}$ Die Weiterentwicklungen der fotografischen Technik vereinfachte die Tätigkeit der Lichtbildner und senkte die Hürde, sich professionell der Fotografie zu widmen. Ende der 188oer Jahre lösten Brom-Gelatinetrockenplatten im Zarenreich das nasse Kollodiumverfahren ab. Fotografen mussten für die Arbeit mit den neuen Fotoplatten kein komplettes Labor mehr mitführen. ${ }^{80}$ Zur gleichen Zeit erleichterte das industriell gefertigte Albuminpapier das

73 Osipov: Katalog Kazanskoj naučno-promyšlennoj vystavki, S. 7.

74 GATO f. 51, op. 37, d. 1440.

75 Elliot: The Photograph in Russia, S. 12; Šipova: Fotografy Moskvy (2006), S. 13-14.

76 Beispielsweise in Tula: GATO f. 9o, op. 1 t 47, d. 4146o, l. 47, 6o, 78; für Moskau: Popov: Iz istorii rossijskoj fotografii, S. 18-20; für St. Petersburg: Ebba Norkute: Der Photograph und seine Majestät, das Theater. Die Photographische Sammlung des Museums für Theater und Musik, Leningrad, in: Nikolaj Nikolaevič Rachmanov (Hrsg.): Das Russland der Zaren. Photographien von 1839 bis zur Oktoberrevolution, Berlin 1989, S. 132-142, S. 140-141.

77 Christopher Stolarski: Another Way of Telling the News. The Rise of Photojournalism in Russia, 1900-1914, in: Kritika, Jg. 12/2011, Heft 3, S. 561-590, S. 572.

78 Sally West: I Shop in Moscow. Advertising and the Creation of Consumer Culture in late Tsarist Russia, DeKalb 2011, S. 37.

79 Leider kann eine solche Untersuchung im Rahmen der vorliegenden Arbeit nicht geleistet werden. Eine Analyse dieser Zusammenhänge wäre jedoch wünschenswert.

8o Barchatova: Russkaja svetopis', S. 210. 
Herstellen fotografischer Abzüge. ${ }^{81}$ Beide Entwicklungen halfen den Lichtbildnern, Aufnahmen herzustellen, und erweiterten die Palette fotografischer Motive.

In Ateliers arbeiteten neben dem Fotografen und seinen Lehrlingen auch Retuscheure und Personen, die darauf spezialisiert waren, Negative abzuziehen. In der Regel waren in einem Studio deutlich mehr Angestellte tätig, die die Bilder bearbeiteten und mit dem Aufnahmeprozess selbst nichts zu tun hatten (teilweise bis zu sechs Personen), als Fotografen (meist nur einer). ${ }^{82}$ Dies verdeutlicht, dass neben der Betätigung des Auslösers der Kamera in einem Studio zahlreiche Aufgaben anfielen. Die Tätigkeit des Fotografen war jedoch die am besten bezahlte innerhalb der Betriebe.

Lichtbildner nutzten für die Herstellung von Fotografien in den ersten Jahrzehnten nach 1839 vornehmlich aus Westeuropa importierte technische Ausstattungen und Chemikalien. Erst in den 188oer Jahren begannen russische Betriebe, sich auf diese Branche und innerhalb derselben zu spezialisieren: Einzelne Unternehmen konzentrierten sich ausschließlich darauf, Negative zu entwickeln. ${ }^{83}$ Auch der Handel mit fotografischen Geräten und Zubehör professionalisierte sich bis zur Jahrhundertwende. Russische Fotoliebhaber hatten zunächst ihre Ausrüstung und die von ihnen benötigten Chemikalien in lokalen Apotheken erstanden, ab den 189oer Jahren öffneten in vielen Städten spezialisierte Foto-Handelsdepots ihre Türen. ${ }^{84} 1882$ nahm Al'bert Feliš $(1837-1908)^{85}$ in St. Peterburg die erste Fabrik für Gelatineemulsion in Betrieb und markierte damit den Beginn der russischen Fotoindustrie. ${ }^{86}$ Allerdings entwickelte sich dieser Wirtschaftszweig nur langsam, und bis zum Ersten Weltkrieg konnte die russische Produktion nie die Nachfrage des russischen Marktes befriedigen. Im Jahr 1896 waren noch immer 85 Prozent aller Materialien, die Fotografen für ihre Arbeit benötigten, Importe aus

81 Problematisch war für die Produktion von Albuminpapier das regelmäßige Auftreten der Hühnerpest, denn pro Tag wurden mehr als 10.000 Eiweiß benötigt. Rolf Sachsse: Fotografie. Vom technischen Bildmittel zur Krise der Repräsentation, Köln 2003, S. 53.

82 Osipov: Katalog Kazanskoj naučno-promyšlennoj vystavki, S. 7, 9.

83 Saburova: Early Masters of Russian Photography, S. 36.

84 Barchatova: Wissenschaft? Handwerk? Kunst!, S. 22.

85 Zwar nennt Barchatova dem Namen "Al'fred Feliš“, doch unterschiedliche fotografische Zeitschriften sprechen von „Al'bert Feliš“. Barchatova: Russkaja svetopis', S. 209; o. A.: Jubilej A. Ė. Feliša, in: Fotograf-Ljubitel', 1891, No. 3, S. 95; o. A.: Nekrolog A. Ė. Feliša, in: Fotografičeskie Novosti, 1908, No. 7, S. 119.

86 Gelatineemulsionen wurden unter anderem benötigt, um fotografische Abzüge anzufertigen. Barchatova: Russkaja svetopis', S. 209. 
Deutschland, Frankreich oder Großbritannien. Später entwickelten sich auch die USA zu einer wichtigen Bezugsquelle in diesem Bereich. ${ }^{87}$

Die Verzahnung der russischen mit der ausländischen Wirtschaft stellte für die Unternehmen im Zarenreich einerseits Vorteile dar, andererseits barg sie Risiken. Um die Jahrhundertwende waren 37 Prozent des russischen Aktienkapitals in ausländischer Hand:88 Die russische Wirtschaft war bereits stark mit dem Weltmarkt verbunden. Vorteile waren zusätzliches finanzielles und unternehmerisches Engagement ausländischer Industrieller. Die negativen Auswirkungen zeigten sich um 1900, als der weltweite Konjunkturabschwung die Wirtschaft des Zarenreichs hart traf. ${ }^{89}$ Mehrere russische Handelshäuser mussten aufgrund einer internationalen Finanzkrise und eines Überangebots an Getreide Konkurs anmelden, im Zuge dessen mussten weitere 3.00o Unternehmen ihren Betrieb einstellen. Zu selben Zeit war der erste Bauabschnitt der Transsibirischen Eisenbahn abgeschlossen worden, so dass die staatlichen Aufträge und die damit verbundene finanzielle Unterstützung der Industrie fehlten..$^{90}$ Während die Konsumgüterindustrie dank der internen Nachfrage vergleichsweise gut durch die Krise kam, waren Metall- und Textilindustrie sehr stark von den Auswirkungen betroffen. ${ }^{91}$

In diese wirtschaftlich schwierige Periode fiel zusätzlich eine Missernte und verschlechterte die Lebensbedingungen der Landbevölkerung merklich. Gleichzeitig stieg die Bevölkerung im Zarenreich generell an, ${ }^{92}$ und immer mehr Menschen strömten in die urbanen Zentren. Mitte des 19. Jahrhunderts

87 Elliot: The Photograph in Russia, S. 13 .

88 An der Spitze standen französische Investoren. Franzosen und Belgier engagierten sich besonders im Hüttenwesen, Briten waren in der Öl- und Goldförderung aktiv, Franzosen und Deutsche waren Geldgeber im Bereich des Maschinenbaus und der chemischen Industrie, weiter brachten sich Deutsche und Belgier besonders in der jungen Branche der Elektroindustrie finanziell ein. Neutatz: Träume und Alpträume, S. 78. Es handelte sich dabei um Investitionen, deren Gewinne nicht ins Ausland abflossen (wie in Beispielen aus der Kolonialwirtschaft), sondern direkt der russischen Wirtschaft zugutekamen. John P. McKay: Ausländische Unternehmer im zarischen Rußland 1860-1914, in: Dittmar Dahlmann; Carmen Scheide (Hrsg.): „... das einzige Land in Europa, dass eine grosse Zukunft vor sich hat". Deutsche Unternehmen und Unternehmer im Russischen Reich im 19. und frühen 20. Jahrhundert, Essen 1998, S. 65-86, S. 65-69.

89 Michail Porfir'evič Vjatkin: Gornozavodskij Ural v 1900-1917 gg., Moskva, Leningrad 1965, S. 24-36.

9o Heiko Haumann: Kapitalismus im zaristischen Staat 1906-1917. Organisationsformen, Machtverhältnisse und Leistungsbilanz im Industrialisierungsprozeß, Königstein 1980, S. 23; Gatrell: Reconceptualizing Russia's Industrial Revolution, S. 231-232; Neutatz: Träume und Alpträume, S. 78.

91 Gatrell: The Tsarist Economy, S. 171-172.

92 Portal: The Industrialization of Russia, S. 811. 
lebten in St. Petersburg etwa 485.00o Menschen, in Moskau etwa 337.000. In den folgenden 30 Jahren wuchsen die Einwohnerzahlen um mehr als das Doppelte - in den 188oer Jahren wurden beide zu Millionenstädten. Bis 1915 hatten St. Petersburg mit 2.318.60o und Moskau mit 1.984.00o die Anzahl ihrer Bewohner ein weiteres Mal in etwa verdoppelt. ${ }^{93}$ Die Städte waren auf dieses Bevölkerungswachstum nicht ausgerichtet, es entstand ein großer Mangel an Wohnraum ${ }^{94}$ und viele Menschen litten unter katastrophalen hygienischen Bedingungen. ${ }^{95}$ Die beiden russischen Metropolen besetzten mit ihren Mortalitätsraten im Vergleich mit anderen europäischen Hauptstädten die Spitzenpositionen..$^{96}$ Die sozialen Probleme und Spannungen der russischen Gesellschaft verschärften sich im Zuge der Wirtschaftskrise weiter, was die Ausbildung extremer politischer Strömungen sowie terroristischer Aktivitäten im Zarenreich förderte. ${ }^{97}$ Die erste Dekade des 20. Jahrhunderts war durch nationale wie internationale Konflikte geprägt: 1902 kam es zu Bauernunruhen,

93 Timothy Colton: Moscow, Governing the Socialist Metropolis, Cambridge u. a. 1995, S. $757-758$.

94 James H. Bater: St. Petersburg. Industrialization and Change, London 1976, S. 173-181, 326342; Schlögel: Jenseits des Großen Oktober, S. 57-64; Clemens Zimmermann: Die Zeit der Metropolen, Urbanisierung und Großstadtentwicklung, Frankfurt a. M. 1996, S. 3o. Zu Wohnverhältnissen siehe auch: Goehrke: Auf dem Weg in die Moderne, S. 317. In Moskau lebten Schätzungen zufolge durchschnittlich 8,7 , in St. Petersburg 7,4 Menschen in einer Wohnung. Sie lagen damit weit über den Zahlen anderer europäischer Hauptstädte wie Paris $(4,3)$, Wien $(4,2)$ oder Berlin $(3,9)$. Blair Ruble: Second Metropolis. Pragmatic Pluralism in Gilded Age Chicago, Silver Age Moscow, and Meiji Osaka, Cambridge u. a. 2001, S. 266.

95 Colton: Moscow, S. 57. Eine eindringliche Schilderung der Missstände gibt auch: Alexander Martin: Der Schmutz, der Gestank und die Stadt. Repräsentationen städtischer Gesellschaft in Moskau 1770-1880, in: Jörg Baberowski; David Feest; Christoph Gumb (Hrsg.): Imperiale Herrschaft in der Provinz. Repräsentationen politischer Macht im späten Zarenreich, Frankfurt a. M., New York 2008, S. 359-403, S. 363-364. Zu Krankheiten in Moskau siehe: Kyoo-Sik Lee: Das Volk von Moskau und seine bedrohte Gesundheit. Öffentliche Gesundheitspflege in Moskau, 1850-1914, Frankfurt a. M. u. a. 1996.

96 Zimmermann: Die Zeit der Metropolen, S. 95.

97 Siehehierzu das Themenheft der Jahrbücher für Geschichte Osteuropas: Anke Hillbrenner; Frithjof Schenk (Hrsg.): Modern Times? Terrorism in Late Imperial Russia, Themenheft: Jahrbücher für Geschichte Osteuropas, Jg. 58/2010, Heft 2, mit Beiträgen von Sally Boniece, Anke Hillbrenner, Lynn Patyk, Frithjof Schenk und Claudia Verhoeven. Zur Ausbildung des Terrorismus siehe auch: Claudia Verhoeven:The odd Man Karakozov. Imperial Russia, Modernity, and the Birth of Terrorism, Ithaca 2009, S. 10-65; Anke Hillbrenner: Gewalt als Sprache der Straße. Terrorismus als Form politischer Kommunikation im Russischen Reich vor 1917, unveröffentlichtes Manuskript der Habilitationsschrift, Bonn 2014; Felicitas Fischer von Weikersthal; Anke Hilbrenner: Imperien in der Krise. Terrorismus und imperiale Gewalt in Osteuropa vom 19. Jahrhundert bis in die Gegenwart, in: Osteuropa, Jg. 66/2016, Heft 4, S. 3-4. 
1903 erschütterte eine Streikwelle die Industriezentren des Imperiums. ${ }^{98}$ Die Wirtschaft des Zarenreichs hatte sich noch nicht von der Krise erholt, als 1904 der Russisch-Japanische Krieg begann. Die zaristische Regierung versuchte, die angespannte Lage im Inneren des Landes durch Gesetze zum Arbeitsschutz zu befrieden, doch die Bemühungen blieben wirkungslos. Spätestens als am 9. Januar 1905 Regierungstruppen in St. Petersburg das Feuer auf unbewaffnete Demonstranten eröffneten, verspielte die Staatsmacht vollends das Vertrauen der Bevölkerung. ${ }^{99}$ Der Generalstreik im Oktober 1905 führte der Regierung schließlich die Macht der neuen Arbeiterschaft vor Augen und resultierte in politischen Veränderungen. ${ }^{100}$

Die hohen Ausgaben für die Kriegsführung und Kosten, die im Zuge der revolutionären Auseinandersetzungen 1905 bis 1907 entstanden, waren so hoch, dass die russische Regierung kurz vor der Zahlungsunfähigkeit stand. Ihre Schulden konnte sie nur dank französischer Kredite decken. ${ }^{101}$ Als Folge der schwierigen finanziellen Lage verzichtete Finanzminister Vladimir Nikolaevič Kokovcov (1853-1943) darauf, die Entwicklung der Industrialisierung nach 1905 von staatlicher Seite weiter zu forcieren. ${ }^{102}$

Die russische Wirtschaft brauchte mehrere Jahre, bis sie sich von der Krise erholt hatte. Erst 1909 begann sie wieder langsam zu wachsen. Hierfür waren Investitionen und Bankkredite, die höher waren als jemals zuvor, sowie der zunehmende Konsum in ländlichen Regionen und Städten verantwortlich. Hohe Getreidepreise steigerten zwischen 1906 und 1912 die Kaufkraft der Bauern. ${ }^{103}$ Die Industrie verzeichnete hohe Wachstumsraten, so dass Russland am Vorabend des Ersten Weltkriegs zur fünftgrößten Wirtschaftsmacht weltweit aufsteigen konnte. ${ }^{104}$ Die Anzahl aller Beschäftigten wuchs dabei

98 Haumann: Kapitalismus im zaristischen Staat, S. 23; Neutatz: Träume und Alpträume, S. 103 .

99 Reginald E. Zelnik: Russian Workers and Revolution, in: Dominic Lieven (Hrsg.): The Cambridge History of Russia, Bd. 2. Imperial Russia, 1689-1917, Cambridge 2006, S. 617636, S. 629-630; Walter Sablinsky: The Road to Bloody Sunday. Father Gapon and the St. Petersburg Massacre of 1905, Princeton 1976, S. 229-271; Gerald D. Surh: 1905 in St. Petersburg. Labor, Society, and Revolution, Stanford 1989, S. 106-115.

100 Hildermeier: Geschichte Russlands, S. 1011-1018.

101 Winks; Neuberger: Europa and the Making of Modernity, S. 235.

102 Ananich: The Russian Economy and Banking System, S. 417-418, 421.

103 Geyer: Der russische Imperialismus, S. 200; Portal: The Industrialization of Russia, S. 845.

104 Dowler: Russia in 1913, S. 27-29. Hildermeier zufolge lassen aktuelle Forschungen zum russischen Wirtschaftswachstum jedoch keine besonderen Auffälligkeiten erkennen. Unter Einbeziehung der Landwirtschaft war die Zunahme höher als die deutsche, britische oder italienische, sie lag jedoch unter der der USA oder Kanadas. Russlands Industrie wuchs nicht ungewöhnlich rasch. Hildermeier: Geschichte Russlands, S. 1148. 
schneller als die Zahl der Fabriken, was darauf hindeutet, dass die bereits bestehenden Unternehmen immer größer wurden. ${ }^{105}$ Obwohl viele dieser Großbetriebe aus der Eisen- und Stahlindustrie waren, entfielen nur 14 Prozent der wertmäßigen Produktion und 21 Prozent der Beschäftigten auf diese Branche. Führend waren Textil- und Nahrungsmittelindustrie, die die Hälfte der wertmäßigen Produktion beziehungsweise 43 Prozent der Arbeiter ausmachten. Das Kapitalwesen war 1913 im Zarenreich gut ausgebaut, wobei 44 Prozent des Geldes in den Banken aus dem Ausland stammte. ${ }^{106}$ Trotz seiner insgesamt guten Wirtschaftslage ging das Zarenreich jedoch mit einer großen Kreditlast von neun Billionen Rubel Schulden in den Ersten Weltkrieg. ${ }^{107}$ Das Land hatte begonnen, seine Schulden abzubauen, doch vor dem Hintergrund des Krieges verpufften diese Anstrengungen. ${ }^{108}$

Die Branche der Fotografie überstand in Russland die Wirtschaftskrise der Jahrhundertwende vergleichsweise gut. Neue Drucktechniken hatten neue Verwendungsmöglichkeiten für Fotografien entstehen lassen: als Abbildungen in Illustrierten oder auf Bildpostkarten. Die erweiterten Reproduktionsmöglichkeiten verschärfte die Frage des Urheberrechts an fotografischen Aufnahmen. ${ }^{109}$ Diese Thematik diskutierten Fachkreise bereits seit dem letzten Drittel des 19. Jahrhunderts, eine zufriedenstellende Lösung hatte die russische Regierung jedoch nicht gefunden. Eine Ursache für die fehlende Gesetzgebung war die weit verbreitete Ansicht, es handle sich bei Fotografien um technische, nicht um künstlerische Bilder. Der Fotograf stelle nur die Kamera auf und bereite die Chemikalien vor. Für die eigentliche Bildherstellung sei die Sonne verantwortlich. ${ }^{110}$ Die Thematik des Urheberrechts war

1051913 waren 54 Prozent aller Beschäftigten in der Industrie in nur fünf Prozent der Betriebe angestellt, die der Fabrikinspektion unterlagen. Hildermeier: Geschichte Russlands, S. 1151-1152.

106 Dowler: Russia in 1913, S. 40-42. Zum Bankensystem: Portal: The Industrialization of Russia, S. 849 .

107 Davon waren 4,3 Billionen Rubel ausländische Schulden und damit mehr als doppelt so viel wie die Summe aller ausländische Investitionen im Zarenreich. Ananich:The Russian Economy and Banking System, S. 421.

108 Dowler: Russia in 1913, S. 27.

$109 \mathrm{Zu}$ Beispielen für illegale Kopien und Verwendungen von Fotografien siehe: Popov: Iz istorii rossijskoj fotografii, S. 23-24. Allgemeineren Überlegungen zur Entwicklung des Urheberrechts siehe bei: Monika Dommann: Autoren und Apparate. Die Geschichte des Copyrights im Medienwandel, Frankfurt a. M. 2014.

110 Barchatova: Wissenschaft? Handwerk? Kunst!, S. 27; Katherine Hill Reischl: Photography and the Crisis of Authorship. Tolstoy and the Popular Photographic Press, in: Jahrbücher für Geschichte Osteuropas, Jg. 6o/2012, Heft 4, S. 533-549, S. 536. 
eng mit der Frage verbunden, ob Fotografien Kunstwerke seien oder nicht."11 Lichtbildner wie Andrej Osipovič Karelin (1837-1906), Sergej L'vovič Levickij oder Sergej Michajlovič Prokudin-Gorskij (1863-1944) engagierten sich für eine gesetzliche Regelung. 1911 verabschiedete die Duma schließlich das erste Gesetz zum Urheberrecht in der russischen Fotografie. ${ }^{112}$ Es sprach den Fotografen für zehn Jahre das alleinige Nutzungsrecht zu, solange es sich nicht um Auftragsarbeiten handelte. In diesem Fall erhielt der Auftraggeber die Verwendungsrechte für die Bilder.113 Damit war offiziell der Umgang mit Fotografien festgeschrieben, allerdings kam es immer wieder zu Verstößen gegen die Regelungen. ${ }^{114}$

Die Berufsausbildung war ein weiterer Bereich im fotografischen Gewerbe, der bis zum Ende des Zarenreichs wenig institutionalisiert und ohne einheitliche Standards blieb. Obwohl die Mitglieder der fotografischen Gesellschaften dieses Thema bereits vor der Jahrhundertwende diskutiert hatten, entwickelte sich abgesehen von Initiativen Einzelner kein Konzept für die Berufsqualifizierung. ${ }^{115}$ Dies führten Fachleute 1910 als ein Grund an, warum die russischen Fotografen auf internationalen Ausstellungen am Vorabend des Ersten Weltkriegs vergleichsweise schlecht abschnitten. In Russland gab es weder Schulen noch Hochschulen, an denen Aufnahmetechniken hätten erlernt werden können. ${ }^{116}$ Möglicherweise galt die Fotografie als zu wenig künstlerisch, als dass sie in die Kunstausbildung Aufnahme gefunden hätte. ${ }^{117}$ Stattdessen erfolgte die Ausbildung zumeist bei einem Meister in dessen Studio. ${ }^{118}$ In den

111 Barchatova: Wissenschaft? Handwerk? Kunst!, S. 19; Hill Reischl: Photography and the Crises of Authorship, S. 548.

112 Popov: Iz istorii rossijskoj fotografii, S. 24-28.

113 Polnoe sobranie zakonov Rossijskoj imperii, Tom 31, 1911, čast' 1, zakon No. 34935 (11.03.1911), S. 194-201, Ob“ avtorskom" prave, S. 201.

114 Popov: Iz istorii rossijskoj fotografii, S. 23.

115 R. Allshouse: Introduction, in: ders. (Hrsg.): Photographs for the Tsar. The Pioneering Colour Photography of Sergei Mikhailovich Prokudin-Gorskii, Commissioned by Tsar Nicholas II., London 1980, S. ix-xxiii, S. x; Barchatova: Russkaja svetopis', S. 231-232; Barchatova: Wissenschaft? Handwerk? Kunst!, S. 27; Stigneev: Vek fotografii, S. 11.

116 Zwar hatten 1896 die Teilnehmer des ersten Kongresses der russischen Vertreter der Fotografie beschlossen, eine Fachschule für Fotomechanik einzurichten, dieser Beschluss wurde jedoch nie verwirklicht. Teilweise boten einzelne Fotografen und fotografische Gesellschaften nach der Jahrhundertwende fototechnische Kurse an, diese fanden jedoch nicht zentral an einer Institution statt. Barchatova: Wissenschaft? Handwerk? Kunst!, S. 27, 36; Barchatova: Russkaja svetopis', S. 231; A. E. Janovskij: Pečatnoe delo, knižnaja torgovlja i knigochranilišča, in: V. I. Kovalevskago (Hrsg.): Rossija v konce XIX veka, Sankt-Peterburg 1900, S. 889-915, S. 901.

117 Barchatova: Wissenschaft? Handwerk? Kunst!, S. 31.

118 Stolarski: The Rise of Photojournalism, S. 43. 
Beständen des zentralen Fotoarchivs in St. Petersburg finden sich mehrere Fotografien, die sich ausschließlich durch eine minimal verschobene Position der Kamera und variierende Belichtung unterscheiden. ${ }^{119}$ Als Autor der Aufnahmen ist immer „Bulla“ vermerkt. Aufgrund der damaligen fotografischen Technik war es jedoch nicht möglich, die Fotoplatte so schnell zu wechseln. Folglich ist davon auszugehen, dass zwei unterschiedlichen Kameras die Bilder anfertigten. Sehr wahrscheinlich entstanden diese Aufnahmen im Zuge der Ausbildung eines Lehrlings. Ein Lichtbildner aus dem St. Petersburger Atelier Bulla $^{120}$ und ein Lehrling platzierten ihre Kameras nebeneinander und fotografierten dasselbe Motiv. Nach der Entwicklung des Negativs beziehungsweise des Abzugs konnte der Lehrling sein Ergebnis direkt mit dem seines Lehrers vergleichen und die eigene Technik verbessern. ${ }^{121} \mathrm{Ab}$ den 1910er Jahren gab es in Russland vermehrt fototechnische Kurse. ${ }^{122}$ Aber erst unter den Bolschewiki entstand 1918 mit dem Vyščij Institut Fotografii i Fototechniki (Höheres Institut für Fotografie und Fototechnik) die erste höhere Bildungseinrichtung, deren Ziel es war, professionelle Fotografen auszubilden. ${ }^{123}$

Zwischen der Jahrhundertwende und dem Beginn des Ersten Weltkriegs nahm die Popularität der Fotografie nochmals stark zu. Besonders kleinere Handkameras wie die Modelle der Firma Kodak zielten darauf ab, das Fotografieren zu einer Massenbeschäftigung zu machen. Mit 16 bis 168 Rubel und Kindermodellen von 2,5 bis 28 Rubel waren die Fotoapparate zumindest für Mitglieder der russischen Mittelschicht erschwinglich. In ihrer Reklame betonte die Firma die Bedeutung einer Kamera als „besten Chronisten des persönlichen Familienlebens. ${ }^{\text {"24 }}$ Die Werbetexte sprachen darüber hinaus die traditionelle Rolle der Frau als derjenigen an, der die häuslichen Pflichten und die Sorge um das Wohl der Familie zufielen. ${ }^{25}$ Die Grafiken der Werbeanzeigen präsentierten Frauenfiguren mit Fotoapparaten, die fast wie kleine Handtaschen aussahen. ${ }^{126}$ Der Hersteller inszenierte die Apparate ganz anders als dies in fotografischen Zeitschriften üblich war, deren Leser besonders auf technische Details Wert legten. Die Zeichnungen von Freizeit- und

\footnotetext{
119 Beispielsweise: CGAKFF d. 3498 und d. 3499.

120 Zur Geschichte der Familie Bulla siehe auch: Kapitel „Fabriken zum Zeitvertreib? Illustrierte Zeitschriften“, S. 241-282.

121 Vielen Dank an Oksana Igorevna Morozan für diesen Hinweis.

122 Barchatova: Russkaja svetopis', S. 231.

123 Barchatova: Wissenschaft? Handwerk? Kunst!, S. 36; Tchmyreva: The History of Russian Photography, S. 518.

124 Kodak, in: Ogonëk, 01.06.1914, S. 25.

125 Engel: Between the Fields and the City, S. 22-24.

126 Hierzu auch: Stolarski: Another Way of Telling the News, S. 564.
} 
Urlaubsszenen, in denen die Kamera zum Einsatz kam, versprachen leicht zu bedienende Geräte. ${ }^{127}$ Sie inszenierten die Fotoapparate als einen allgemein zugänglichen und erstrebenswerten Konsumartikel. Es bleibt allerdings unklar, in wie weit der Versuch der Firma Kodak, die Amateurfotografie zu einem Massenphänomen im Zarenreich zu machen, wirklich Erfolg hatte. Stigneev betont in seiner Darstellung zwar, dass dies der Fall gewesen sei, und führt als Argument an, dass in den Jahren vor dem Ersten Weltkrieg jährlich 25.0oo Fotoapparate importiert worden seien und Fotografen bis zu 70 Millionen Fotoplatten entwickelten. ${ }^{128}$ Jedoch ist fraglich, ob bei einer Anzahl von 25.000 Geräten gegenüber einer Gesamtbevölkerung von 165,7 Millionen von einem Massenphänomen gesprochen werden kann. Sicher ist, dass die Fotografie vor dem Ersten Weltkrieg für viele Menschen im Zarenreich zu einem Bestandteil des Alltags geworden war.

\section{Die Zukunft der russischen Wirtschaft}

Welchen Weg sollte das Zarenreich in die Zukunft wählen? Diese Frage trieb Mitglieder der führenden Gesellschaftsschichten während des 18. und besonders des 19. Jahrhunderts um. Die Zeitgenossen führten Diskussionen darüber, welche politische Rolle das russische Imperium in der Welt übernehmen, wie es sich in Fragen der Kultur oder der Nation gegenüber Westeuropa positionieren sollte und welches Wirtschaftsmodell am besten zum Zarenreich passe. ${ }^{129}$ Diese Kontroversen bilden einen wichtigen Kontext, vor dem die fotografischen Aufnahmen der Industrialisierung entstanden sind und in dem sie interpretiert werden müssen. Ob Russland seine Wirtschaft industrialisieren solle und falls ja, in welcher Form, war eines der zentralen Themen dieser Debatten. ${ }^{130}$ Im Folgenden werden die wichtigsten Positionen der Befürworter und Gegner einer Industrialisierung kurz skizziert, wobei der Fokus auf Fragen zur ökonomischen Entwicklung Russlands liegt. Es kommen

127 Kodak, in: Ogonëk, 02.06.1914, S. 25; Kodak, in: Ogonëk, 15.o6.1914, S. 23.

128 Stigneev: Vek fotografii, S. 15. Gerade im Zarenreich, wo es nur eine kleine Mittelschicht gab, scheint es wenig wahrscheinlich, dass so viele einfache Menschen sich eine Kamera leisten konnten. Auch in Deutschland und Österreich-Ungarn war Ende des 19. Jahrhunderts die Zahl der Amateure noch überschaubar. Starl: Im Prisma des Fortschritts, S. 63 .

129 Auch in Westeuropa entzündeten sich am Ort und Gegenstand der Fabrik und seiner wirtschaftlichen wie symbolischen Bedeutung Diskussionen über die Zukunft der Wirtschafts- und der Gesellschaftsformen. Hierzu: Boch: Fabriken, S. 537-539.

130 Blackwell: The Beginnings of Russian Industrialization, S. 146; Chromov: Ėkonomičeskoe razvitie Rossii, S. 147-148. 
ausschließlich Vertreter der alphabetisierten Eliten des Zarenreichs zu Wort; es ist wenig darüber bekannt, wie einfache russische Arbeiter zu unterschiedlichen Zeiten über die Industrialisierung dachten. Ihre Stimme fand kaum Gehör in den gesellschaftlichen Diskussionen um die wirtschaftliche Zukunft Russlands.

Bezugsrahmen für die Debatten über die Zukunft des Zarenreichs bildete schon seit dem 15. Jahrhundert Westeuropa. ${ }^{131}$ Die Frage, ob sich Russland anpassen oder auf seine Andersartigkeit setzen sollte, beschäftigte über Jahrhunderte die Elite des Reichs. Einen ersten Höhepunkt erlebten die Auseinandersetzungen, nachdem Peter I. das Zarenreich in großangelegten Reformen sowie durch die Eroberung des Zugangs zur Ostsee und der Gründung St. Petersburgs stärker an Westeuropa herangerückte hatte; nicht allen erschien das westeuropäische Vorbild erstrebenswert. ${ }^{132}$ Die politische Elite des Zarenreichs blieb jedoch auf dem von Peter dem Großen vorgegebenen Kurs. Seine Nachfolger auf dem Zarenthron orientierten sich ebenfalls in vielen Bereichen an westeuropäischen Staaten und setzten zahlreiche wirtschaftliche, rechtliche, politische und kulturelle Veränderungen per Dekret von oben durch. Viele ihrer Beschlüsse kollidierten allerdings mit den Gegebenheiten vor Ort und scheiterten an der russischen Realität. ${ }^{133}$ Diese positiven wie negativen Erfahrungen verarbeiteten russische Intellektuelle in ihren Diskussionen über die Zukunft des Zarenreichs.

Nach dem Wiener Kongress (1814-1815) intensivierten sich die Auseinandersetzungen, ob Russland sich stärker einer Mechanisierung seiner Industrie und neuen, kapitalistischen Produktionsmethoden zuwenden sollte. Große Gegner dieses Zukunftsmodells waren Viktor Pavlovič Kočubej (1768-1834), der von 1802 bis 1807 und von 1819 bis 1823 russischer Innenminister war, sowie Egor Francevič Kankrin (1774-1845), ab 1823 bis 1844 russischer Finanzminister. Beide argumentierten, dass das Russische Reich ein Agrarland bleiben solle und allein die bäuerliche Industrie zu fördern sei. ${ }^{134}$ Gleichgesinnte führten die Situation der englischen Arbeiter als Negativbeispiel an und argumentierten, dass es den russischen Bauern in

\footnotetext{
131 Schenk: Russlands Aufbruch in die Moderne?, S. 187.

132 Alexander Gerschenkron: Europe in the Russian Mirror. Four Lectures in Economic History, Cambridge 1970, S. 3; Andrew Baruch Wachtel; Ilya Vinitsky: Russian Literature, Cambridge 2009, S. 92.

133 Effi Böhlke: Endzeit. Zukunftsvorstellungen im russischen politisch-philosophischen Denken um 1900, in: Sönke Neitzel (Hrsg.): 1900. Zukunftsvisionen der Großmächte, Paderborn u. a. 2002, S. 31-54, S 32; Neutatz: Träume und Alpträume, S. 52.

134 Blackwell: The Beginnings of Russian Industrialization, S. 140-144.
} 
der Landwirtschaft besser gehe als in entmenschlichten Fabriken. ${ }^{135}$ Sollte es überhaupt zu einer Industrialisierung des Russischen Reichs kommen, forderten Nationalisten und Traditionalisten, dass sich deren Geschwindigkeit an der Entwicklung der Landwirtschaft orientieren müsse. ${ }^{136}$ Vertreter der zu Beginn des 19. Jahrhunderts neu in Russland entstandenen Wirtschaftswissenschaften, ${ }^{137}$ beispielsweise Heinrich Friedrich von Storch (17661835), argumentierten ähnlich. Allerdings meldeten sich auch Stimmen zu Wort, die eine schnelle Industrialisierung des Zarenreichs forderten. ${ }^{138}$ Die Befürworter einer raschen Industrialisierung befürchteten, Russland könnte von Europa abgehängt werden, wenn es sich wirtschaftlichen Veränderungen verschlösse. Sie forderten die Unterstützung der russischen Regierung mittels protektionistischer Politik. Bliebe diese aus, seien Stagnation, Inflation und Armut die Folgen - die Gruppe der nach Westen orientierten Protagonisten zitierte die schwierige Lage Spaniens als abschreckendes Beispiel. ${ }^{139}$ Beide Lager waren jedoch keine homogenen Gruppen und umfassten Vertreter unterschiedlicher Ansichten, deren Standpunkte sich teilweise überschnitten. ${ }^{140}$

Ein wichtiger Bezugspunkt, sowohl für die Befürworter einer am Vorbild Westeuropas ausgerichteten Entwicklung (zapadniki - die Westler) als auch für ihre Gegner (die Slavophilen), stellte der 1836 auf Russisch publizierte Erste Philosophische Brief Pëtr Jakovlevič Čaadaevs (1794-1856) dar. Čaadaev widmete sich in seinem Schreiben dem Verhältnis von Russland und Europa und beklagte, dass Russland Europa nur nachgeahmt, aber

1351845 veröffentliche Friedrich Engels seine Abhandlungen über die Lebensbedingungen der englischen Arbeiter. Friedrich Engels: Die Lage der arbeitenden Klasse in England. Nach eigner Anschauung und authentischen Quellen, Leipzig 1845.

136 Blackwell: The Beginnings of Russian Industrialization, S. 123, 126.

137 Die Wirtschaftswissenschaft hatte im Zarenreich keine lange Tradition. Darum hatten die Ideen westeuropäischer Ökonomen unter anderen um die Mitte des Jahrhunderts auch Marx und Engels einen besonders starken Einfluss auf die russischen Wissenschaftler. Esther Kingston-Mann: In Search of the True West. Culture, Economics, and Problems of Russian Development, Princeton 1999, S. 82.

138 Blackwell: The Beginnings of Russian Industrialization, S. 145; Gerschenkron: Europe in the Russian Mirror, S. 7. Zu den Debatten innerhalb der russischen Wirtschaftswissenschaften siehe auch: Barnett: A History of Russian Economic Thought.

139 Blackwell: The Beginnings of Russian Industrialization, S. 124. Zur wirtschaftlichen Situation in Spanien siehe beispielsweise: Walther L. Beckmann; Horst Pietschmann: Geschichte Spaniens. Von der frühen Neuzeit bis zur Gegenwart, Stuttgart ${ }^{4}{ }_{2005}$, S. $283^{-}$ 289; Gabriel Tortella: The Development of Modern Spain. An Economic History of the Nineteenth and Twentieth Centuries, Cambridge, Mass. u. a. 2000, S. 73-114.

Blackwell: The Beginnings of Russian Industrialization, S. 146. 
nichts Eigenständiges hervorgebracht habe. ${ }^{141}$ Die Slavophilen bedienten sich Čaadaevs Argumentation, um zu beweisen, dass die Gruppe der Westler sowie ihre Ideen nicht russisch seien und folglich nicht in der Lage, ein für das Zarenreich passendes Zukunftskonzept zu entwerfen. ${ }^{142}$ Sie selbst lehnten vermehrte Gründungen von Fabriken ab, denn sie befürchteten, dass ein Wachstum der Industrie auf Kosten der Gesundheit und Bildung des Volkes gehen könne und die Fabrikarbeit die Menschen abhängig machen würde. Ein erwerbsloser Arbeiter sei nicht mehr in der Lage, sich selbst zu ernähren. ${ }^{143}$ Außerdem lehnten sie das Konzept des Kapitalismus ab, weil es ihren Werten und Vorstellungen von Gesellschaft widersprach. ${ }^{144}$ Trotz ihrer Kritik wandten sich die Slavophilen nicht von Westeuropa ab. Sie sahen Russlands Aufgabe vielmehr darin, Westeuropa mit den sozialen Problemen zu helfen, die die Industrialisierung hatte entstehen lassen. Sie schlugen die kollektiv und paternalistisch organisierte russische obščina (Dorfgemeinschaft) als Alternativmodell zu einer auf Individualität ausgerichteten westeuropäischen Gesellschaft vor. ${ }^{145}$

Die Diskussionen zwischen Westlern und Slavophilen ging weit über den Bereich der Ökonomie hinaus und erreichten insbesondere mit Literatur und Literaturkritiken ein breites Publikum. ${ }^{146}$ Der Literaturkritiker Vissarion Grigor'evič Belinskij (1811-1848), der ein wichtiger Vertreter des prowestlichen Lagers war, griff ebenfalls Čaadaevs Argumentation auf. Er vertrat die Ansicht, dass es eine wichtige Fähigkeit sei, sich eine Kultur anzueignen und in etwas Neues umzuformen. Diese wichtige Kompetenz erlaube Russland, zur Nation

141 Petr Jakovlovič Čaadaev: Erster Philosophischer Brief, in: Dmitrij Tschižewskij; Dieter Groh (Hrsg.): Europa und Russland. Texte zum Problem des westeuropäischen und russischen Selbstverständnisses, Darmstadt 1959, S. 73-93; Böhlke: Endzeit, S. 32-34.

142 Böhlke: Endzeit, S. 13.

143 Tugan-Baranovskij: Geschichte der russischen Fabrik, S. 328-330.

144 Andrzej Walicki: The Slavophile Controversy. History of a Conservative Utopia in Nineteenth-Century Russian Thought, Oxford 1975, S. 450.

145 Susanna Rabow-Edling: Slavophile Thought and the Politics of Cultural Nationalism, Albany 2006, S. 21. Die Rolle und Bedeutung der Bauern war ein, wenn nicht der zentrale Streitpunkt in den Auseinandersetzungen zwischen Westlern und Slavophilen. Während die Slavophilen die russische Landbevölkerung ins Zentrum ihrer Gesellschaftskonzepte rückten, sahen die Westler die Dorfbevölkerung als Menschen, die zunächst zivilisiert werden müssten, bevor sie eine gesellschaftliche Funktion einnehmen könnten. Kingston-Mann: In Search of the True West, S. 85.

146 Walicki: The Slavophile Controversy, S. 413. Literaten brachten sich bereits im 18. Jahrhundert in politische Diskussionen ein und galten im Zarenreich als die „besseren Politiker“. Ingrid Schierle: Semantiken des Politischen im Russland des 18. Jahrhunderts, in: Wilibald Steinmetz (Hrsg.): Politik. Situationen eines Wortgebrauchs im Europa der Neuzeit, Frankfurt a. M., New York 2007, S. 226-247, S. 247. 
$\mathrm{zu}$ werden und gleichzeitig einen Beitrag zur Weltkultur zu leisten. ${ }^{147}$ Die menschliche Vernunft und der Einsatz von Maschinen ermöglichten es dem Menschen, sich von der Natur unabhängig zu machen, und ebneten den Weg in eine bessere Zukunft. ${ }^{148}$ Insgesamt waren die Standpunkte im Lager der Westler uneinheitlicher als die der Slavophilen. Problematisch war für Intellektuelle, die wie Belinskij oder Aleksandr Ivanovič Gercen (1812-1870) sozialistischen Ideen nahestanden, dass die Industrialisierung eng mit der Verbreitung des Kapitalismus zusammenhing. ${ }^{149}$ Darum diskutierten sie Entwicklungsmodelle der Industrialisierung, welche die russischen Besonderheiten stärker bewahrten. ${ }^{150}$ Ein solcher Prozess der Rezeption westeuropäischer Ideen und deren Übertragung auf die Gegebenheiten im Zarenreich war charakteristisch für russische Intellektuelle im 19. Jahrhundert. Durch Reisen außerhalb des Zarenreichs, Kontakte zu russischen Exilanten in Westeuropa sowie Freundschaften zu Ausländern waren russische Denker mit den Diskussionen und Themen der europäischen Wissenschaft und Geisteswelt bestens vertraut. Ihre häufig sehr guten Sprachkenntnisse ermöglichten es ihnen, die Werke ihrer ausländischen Kollegen in der Originalsprache zu lesen, und ihre internationalen Netzwerke sorgten dafür, dass Neuerscheinungen bereits nach kurzer Zeit auch in den entsprechenden Kreisen im Zarenreich zirkulierten.

In den 1840er Jahren stieg unter russischen Intellektuellen im Exil sowie innerhalb des Zarenreichs das Interesse an den Arbeiten von Karl Marx (1818-1883) und Friedrich Engels (1820-1895). Bereits mehrere Jahre bevor die russische Zensurbehörde Das Elend der Philosophie von Marx 1848 freigab, ${ }^{151}$ zirkulierten unter kritisch eingestellten Intellektuellen illegale Kopien der Bücher von Marx und Engels. Ihre Ideen beeinflussten die Diskussionen

147 Böhlke: Endzeit, S. 34. Zu Belinskijs Argumentation auch: Walicki: The Slavophile Controversy, S. 398-403; 413-422.

148 Isaiah Berlin: Russische Denker, Frankfurt a. M. 1981, S. 207-250; Kingston-Mann: In Search of the True West, S. 81-87.

149 Während beide ursprünglich stark von europäischen Ideen beeinflusst waren und diese unterstützten, erlebten sie auf ihren Reisen Ende der 1840er Jahre eine starke Desillusionierung. Daraufhin wandelten beide ihre Weltanschauungen nochmals ab. Walicki: The Slavophile Controversy, S. 440-442. Zur Haltung beider Publizisten siehe auch: Derek Offord: Nineteenth-Century Russian Thought and Literature, in: Neil Cornwell (Hrsg.): The Routledge Companion to Russia Literature, London 2001, S. 123-135, S. 128.

150 Vera Toltz: The West, in: William Leatherbarrow, Derek Offord (Hrsg.): A History of Russian Thought, Cambridge u. a. 2010, S. 197-216, S. 210-211.

$15^{1}$ Die Argumentation für diese Entscheidung lautete, dass Marx' Werk sich nicht mit Russland auseinandersetzte und die Abhandlungen in hohem Maße abstrakte Spekulationen seien. W. N. Kotow: Eindringen und Verbreitung der Ideen von Karl Marx und Friedrich Engels in Russland. Von den vierziger Jahren bis in die neunziger Jahre des 19. Jahrhunderts, Berlin (Ost) 1956, S. 14-15. 
der regierungskritischen Oppositionellen stark. Weitere wichtige Impulse für die Diskussion steuerte in der zweiten Hälfte des 19. Jahrhunderts Nikolaj Gavrilovič Černyševskij (1828-1889) bei. ${ }^{152}$ Černyševskij entwickelte die Theorie, dass nicht jedes Land alle Entwicklungsstufen auf dem Weg zum Sozialismus durchlaufen müsse, sondern einzelne Stufen übersprungen werden könnten. ${ }^{153}$ Daraus ergäben sich für das russische Imperium neue Entwicklungsmöglichkeiten. In den 186oer und 1870er Jahren bemühte sich die russische Regierung darum, das Zarenreich gegenüber marxistischen Ideen abzuriegeln, doch diese Versuche waren vergebens. ${ }^{154}$ Insbesondere die 1872 erschienene russische Übersetzung von Marx' Das Kapital ermöglichte auch politisch interessierten Bevölkerungsgruppen abseits der intellektuellen Elite den Zugang zu marxistischen Ideen. ${ }^{155}$

Im Laufe der 1880er Jahre vollzog sich im Zarenreich der Übergang zu einem nachhaltigen, industriellen Wachstum, wobei die Gestaltungsmöglichkeiten für die zukünftige Ausrichtung der russischen Gesellschaft weiter offen zu sein schienen. Die Vorschläge aus unterschiedlichen politischen Lagern zeigten das große Spektrum vorhandener Zukunftskonzepte: Vertreter der Autokratie forderten, die Wirtschaft nach westlichem Vorbild umzugestalten. Allerdings sollten die Reformen ausschließlich eine ökonomische Stoßrichtung haben, das politische System nicht tangieren und ohne Verwendung ausländischen sowie jüdischen Kapitas erfolgen. ${ }^{156}$ Mitglieder der liberalen Opposition drangen neben der forcierten Industrialisierung auf politische Reformen, während die marxistischen Sozialdemokraten das Zarenreich zu einem Industriestaat mit einer starken Arbeiterklasse machen wollten. Die Kritik der russischen Marxisten wandte sich weniger gegen die Ausbreitung eines kapitalistischen Wirtschaftssystems, sondern in erster Linie gegen die in ruralen Gebieten immer noch weit verbreitete Naturalwirtschaft. Dem Kapitalismus gestanden

${ }_{152} \mathrm{Zu}$ Černyševskijs Bedeutung für die russischen Auseinandersetzungen über die Entwicklung der russischen Gesellschaft: Kingston-Mann: In Search of the True West, S. 102-106.

153 Hildermeier: Das Privileg der Rückständigkeit, S. 579-580.

154 Insbesondere über russischen Emigranten in Westeuropa erreichten die Werke und Ideen die russischen Intellektuellen. Kotow: Eindringen und Verbreitung der Ideen von Karl Marx und Friedrich Engels in Russland, S. 26-28.

155 Studenten und Mitglieder von Arbeiterzirkeln diskutierten die Thesen des Kapitals. Kotow: Eindringen und Verbreitung der Ideen von Karl Marx und Friedrich Engels in Russland, S. 30-33. Das Buch hatte im Zarenreich erscheinen können, weil die Zensurbehörde davon ausging, dass die russischen Leser den Inhalt nicht verstünden und Marx sich nur an wenigen Stellen der russischen Wirtschaft zuwende.

${ }_{15} 6$ Zur negative Wahrnehmung ,jüdisch“ geführter Eisenbahngesellschaften beispielweise: Schenk: Russlands Fahrt in die Moderne, S. 315. 
die Marxisten zumindest eine zivilisatorische Funktion zu, mit der die Landbevölkerung modernisiert werden könne, um sie in einem zweiten Schritt zu politischen Akteuren zu erziehen. ${ }^{157}$ Slavophile und Mitglieder nationalistisch konservativer Kreise sprachen sich gegen die Ausbreitung der Industrie aus. Sie fürchteten die Proletarisierung der Arbeiterschaft ${ }^{158}$ und hofften, die russische Gesellschaft in den Zustand vor den Reformen Peters I. zurückzuführen. ${ }^{159}$ Die sozialrevolutionäre Bewegung der Narodniki lehnte die Industrialisierung ebenfalls ab und stützte ihre Hoffnungen und Zukunftsvorstellungen auf die Landbevölkerung als Hort russischer Kultur und Traditionen. Sie sahen die kollektiv organisierte russische Bauerngemeinde (obščina) als potentielle Organisationsform sozialen Zusammenlebens, mit der es möglich werden könne, direkt zu einem sozialistischen Gesellschaftsmodell überzugehen. ${ }^{160}$ Zahlreiche Aktivisten der Narodniki gingen mit dem Ziel in die Dörfer, die Bauern zu politisieren, mussten aber bald desillusioniert feststellen, dass diese wenig Interesse für revolutionäre Ideen aufbrachten. ${ }^{161}$

Zehn Jahre später wurden die unter Intellektuellen geführten Diskussionen um Russlands Zukunft von der Realität eingeholt: Die Entwicklung und Mechanisierung der russischen Wirtschaft war so weit vorangeschritten, dass die Zeitgenossen nicht mehr umhin kamen, sich mit der Präsenz des Kapitalismus im Zarenreich und dessen Folgen auseinanderzusetzten. ${ }^{162}$ Vittes

157 Kingston-Mann: In Search of the True West, S. 143.

${ }_{15} 8 \mathrm{Zu}$ den Ängsten vor einem russischen Proletariat und den Maßnahmen, die eine solche Entwicklung verhindern sollten: Hans Rogger: Russia in the Age of Modernisation and Revolution. 1881-1917, London, New York 1983, S. 109-119.

159 Stephen Lukashevich: Ivan Aksakov, 1823-1886. A Study in Russian Thought and Politics, Cambridge Mass. 1965, S. 10-11.

160 Neutatz: Träume und Alpträume, S. 52-54. Zur Entwicklung der Volkstümler oder Populisten: Andrzej Walicki: A History of Russian Thought from the Enlightenment to Marxism, Stanford 1979, S. 28; Derek Offord: The People, in: William Leatherbarrow; ders. (Hrsg.): A History of Russian Thought, Cambridge u. a. 2010, S. 241-262, S. 252-255.

161 Kingston-Man: In Search of the True West, S. 140-141; Katja Bruisch: Als das Dorf noch Zukunft war. Agrarismus und Expertise zwischen Zarenreich und Sowjetunion, Köln, Weimar, Wien 2014, S. 60-72; Jeong-Sook Hahn: Sozialismus als „bäuerliche Utopie?“. Agrarsozialistische Konzeptionen der Narodniki und Neonarodniki im 20. Jahrhundert in Rußland, Tübingen, Univ., Diss., 1994. Viele Mitglieder der Narodniki sahen Marx' Beschreibung der Bauern im Kapital als Bestätigung für ihre enttäuschenden Erfahrungen mit der russischen Landbevölkerung. Als Reaktion emanzipierten sie sich von Marx, und als dieser 1883 in einem Brief an Vera Ivanovna Zasulič (1849-1919) dafür warb, die russischen Bauern stärker in die politische Entwicklung einzubeziehen, hatten die Revolutionäre bereits beschlossen, sich statt den Bauern der Arbeiterbewegung zuzuwenden.

162 Hildermeier: Das Privileg der Rückständigkeit, S. 589; Dowler: The Intelligentsia and Capitalism, S. 263. 
Wirtschaftspolitik beschleunigte den Prozess der Industrialisierung weiter und entzog den autokratischen Eliten ihre Existenzgrundlage, so dass der Adel um seine gesellschaftliche Führungsrolle bangte. Die Folge waren hitzige öffentliche Auseinandersetzungen, die sich bis in Regierungskreise zogen und eine Konfrontation zwischen Finanz- und Innenministerium zur Folge hatten. ${ }^{163}$

Um die Jahrhundertwende war für die Bewohner der Städte nicht mehr zu übersehen, dass die Industrialisierung Russland erreicht hatte. Gerade St. Petersburg galt den Zeitgenossen als der Ort der Moderne schlechthin, wobei diese Bezeichnung in der öffentlichen Diskussion durchaus ambivalent war. Häufig verwendeten Autoren in diesem Zusammenhang die Vokabeln Krankheit oder Tod, was angesichts der hohen Mortalitätsrate in der Hauptstadt nachvollziehbar erscheint. ${ }^{164}$ Die Verbindung von Tod und Stadt war nicht neu, sie hatte in der westeuropäischen Literatur namhafte Vorbilder, so den Franzosen Charles-Pierre Baudelaire (1821-1867). ${ }^{165}$ Die Angst vor den Veränderungen und Folgen der Industrialisierung war keine ausschließlich russische Erscheinung. ${ }^{166}$ Im europäischen Vergleich war der Pessimismus im Zarenreich jedoch besonders stark, und die öffentlich ausgetragenen Diskussionen hatten eine große politische und soziale Reichweite. Die russischen Intellektuellen sorgten sich, wie das Zarenreich die Herausforderungen der späten und rasanten Industrialisierung meistern könnte. ${ }^{167}$ Darüber hinaus trugen wirtschaftliche Interessen dazu bei, dass Autoren mit einer skeptischen Haltung zur Industrialisierung mehr Aufmerksamkeit und Plattformen fanden: Schockierende Sensationsmeldungen, die ein negatives Bild der Industrialisierung entwarfen, verkauften sich in der Presse gut und waren bei Herausgebern beliebt. ${ }^{168}$

163 Das Innenministerium vertrat die Interessen des gutsbesitzenden Adels, das Finanzministerium stand hinter der Politik Vittes. Löwe: Von der Industrialisierung zur ersten Revolution, S. 205.

164 Matthias Schwartz: Das Ende von Petersburg. Utopie und Apokalypse in der russischen Literatur des Fin de Siècle, in: Zeitschrift für Geschichtswissenschaft, Jg. 63/2015, Heft 11, S. 982-1000, S. 989-994; Mark Steinberg: Petersburg. Fin de Siècle, New Haven, London 2011, S. 1-2, 4. Zimmermann: Die Zeit der Metropolen, S. 95.

165 Steinberg: Petersburg, S. 127.

166 Zur Skepsis gegenüber technischen Neuerungen in Westeuropa siehe: Heßler: Kulturgeschichte der Technik, S. 110-111.

167 Die Opposition der autokratischen Regierung im Zarenreich teilte sich in die Gruppe der bürgerlichen Elite und die der sozialrevolutionären Intelligencija. Neutatz: Träume und Alpträume, S. 52-53; Nathaniel Knight: Was the Intelligentsia Part of the Nation? Visions of Society in Post-Emancipation Russia, in: Kritika, Jg. 7/2006, Heft 4, S. 733-758.

168 Steinberg: Petersburg, S. 1, 9, 13. 
Am Vorabend des Ersten Weltkriegs äußerten sich besonders Mitglieder der konservativen und nationalen Kreise ablehnend gegenüber dem Kapitalismus. Sie entstammten zu großen Teilen dem Landadel und assoziierten den Kapitalismus mit dem Judentum und Westeuropa. Indem sie sich gegen das neue Wirtschaftssystem wandten, hofften sie zu verhindern, dass ausländische Interessen das Zarenreich dominierten. Mühe hatten national Konservative als auch der Zar mit den Vorteilen, die die Industrialisierung insbesondere für die Landwirtschaft bot. Weniger radikale Konservative lehnten zwar die privatwirtschaftliche Großindustrie ab, sprachen sich aber für staatliche Großbetriebe aus und forderten, dass kleinere Unternehmen staatliche Unterstützung erhalten sollten. Neben nationalistisch, konservativ und volkstümlich Gesinnten wollten auch die Liberalen die Bauern vor der Ausbeutung durch die Industrie und vor einer Proletarisierung schützen. Auch Journalisten und Redakteure russischer Zeitungen schlossen sich der Stimmung an und zeichneten in ihren Texten das Bild der Unternehmer in den negativsten Farben. Eine Ausnahme stellten diesbezüglich die Marxisten und marxistisch beeinflussten Sozialdemokraten dar. Für sie war der Kapitalismus ein notwendiger Entwicklungsschritt auf dem Weg zu einer kommunistischen Gesellschaft. ${ }^{169}$

\subsection{Die russische Wirtschaft im Spiegel der Literatur}

Die kreative Intelligenz, wie Wayne Dowler russische Schriftsteller und Künstler bezeichnet, brachte sich ebenfalls in die Auseinandersetzung um die Zukunft der russischen Wirtschaft ein. Auch unter ihnen gab es Befürworter wie Gegner der Industrialisierung. Starke Befürworter der neuen Entwicklungen waren zu Beginn des 20. Jahrhunderts sozialistische Autoren wie der spätere Volkskommissar für Bildung, Anatolij Vasil'evič Lunačarskij (1875-1933) oder Aleksandr Aleksandrovič Bogdanov (1873-1928). Bogdanov zeichnete in seinem Buch Krasnaja zvezda (Roter Stern) die Utopie einer sozialistischen Zukunft, in der sich das Leben in hochmodernen Städten und Fabriken abspielte. ${ }^{170}$

Die Mitglieder des in Italien begründeten Futurismus waren ebenfalls von Technik begeistert und sahen in ihr die Möglichkeit, eine neue

169 Dowler: Russia in 1913, S. 233, 241-255. Beispielweise feierte der politisch links orientierte Publizist und Dichter Aleksej Kapitonovič Gastev (1882-1939) in seinen Texten den technischen Fortschritt in Form von Flugzeugen, U-Booten oder Röntgenstrahlen. Steinberg: Petersburg, S. 38 .

170 Richard Stites: Revolutionary Dreams. Utopian Vision and Experimental Life in the Russian Revolution, New York u. a. 1989, S. 32-33. 
Zukunft zu errichten. Die Haltung der italienischen und russischen Futuristen unterschied sich jedoch stark: Die russischen Anhänger der Bewegung hofften ebenfalls auf einen kulturellen Neubeginn, sie standen Urbanismus und Technikbegeisterung jedoch skeptisch gegenüber. ${ }^{171}$ Vasilij Vasil'evič Kamenskijs (1884-1961) Faszination für das Fliegen ${ }^{172}$ oder die Oper Pobeda na solcem (Sieg über die Sonne) waren Ausnahmeerscheinungen. Mehrheitlich empfanden russische Futuristen die neue Zeit als Bedrohung. ${ }^{173}$ Viktor Vladimirovič Chlebnikov (1885-1922) und Vladimir Vladimirovič Majakovskij (1983-1930) beschrieben in ihrer Lyrik, wie der Einzelne unter der enormen Dynamik der Veränderungen in der Großstadt litt. ${ }^{174}$

Die Skepsis gegenüber der Industrialisierung hatte eine lange Tradition unter russischen Literaten. Häufig spiegelten einzelne literarische Figuren die Haltung der Autoren gegenüber der Industrialisierung. Ivan Sergeevič Turgenev (1818-1883), Ivan Savvič Nikitin (1824-1861), Aleksandr Nikolaevič Ostrovskijs (1823-1886) sowie Anton Pavlovič Čechov (1860-1904) ließen in ihren Werken Karikaturen von Unternehmern vom Lande auftreten, deren ausschließliches Interesse darin bestand, um jeden Preis das eigene Vermögen zu vergrößern. ${ }^{175}$ Diese und ähnliche Vorurteile gegenüber Kaufleuten und Industriellen waren weit verbreitet. ${ }^{176}$ Demgegenüber ist es eine Ausnahme, dass Maksim Gor'kij

171 Susanne von Falkenhausen: Futurismus und andere Avantgarden. Chiffren (national-) revolutionärer Identität in Italien und der frühen Sowjetunion, in: Damian Dombrowski (Hrsg.): Kunst auf der Suche nach der Nation. Das Problem der Identität in der italienischen Malerei, Skulptur und Architektur vom Risorgimento bis zum Faschismus, Berlin 2013, S. 207-228, S. 214-216. Zu den Diskussionen bei Futuristen, Suprematisten und Konstruktivisten: Georg Witte: Archaische Zukunftswesen. Andrej Platonovs Werkzeugmenschen, in: Osteuropa, Jg. 66/2016, Heft 8-10, S. 217-233, S. 218.

172 Scott W. Palmer: Dictatorship of the Air. Aviation Culture and the Fate of Modern Russia, Cambridge 2006; Robert Kluge: Der sowjetische Traum vom Fliegen. Analyseversuch eines gesellschaftlichen Phänomens, Leipzig 1997.

173 Hans Günther: Kontrastbilder. Futurismus in Italien und Rußland, in: Christa Ebert (Hrsg.): Kulturauffassungen in der literarischen Welt Rußlands. Kontinuitäten und Wandlungen im 20. Jahrhundert, Berlin 1995, S. 140-158, S. 142-144.

174 Reinhard Lauer: Geschichte der russischen Literatur. Von 1700 bis zur Gegenwart, München 200o, S. 495-508; Felix Philipp Ingold: Der große Bruch. Rußland im Epochenjahr 1913, Kultur, Gesellschaft, Politik, München 2000, S. 179-187.

175 Heller: Geschichte des modernen Unternehmertums, S. 2-3.

${ }_{176}$ Zeitgenössische Kaufleute nahmen die negative Haltung ihnen gegenüber sehr wohl wahr und bemühten sich vor dem Ersten Weltkrieg selbstbewusst auf die Vorurteile zu reagieren. Der Kaufmann Pavel Pavlovič Rjabušinskij erklärt 1912 auf dem Bankett anlässlich des 100. Jahrestags der Gründung der Firma Konovalovs: „Es ist an der Zeit, daß die russische Kaufmannschaft ihren Platz als der führende Stand Rußlands einnimmt, es ist an der Zeit, mit Stolz den Titel des russischen Kaufmanns zu tragen, anstatt hinter 
(1868-1936) den Holzhändler Antipa Ivanovič Sykov in seinem Theaterstück Zykovy (die Zykovs) als überwiegend positiv darstellte. ${ }^{177}$

Um die Mitte des 19. Jahrhunderts entwarfen russische Autoren ein Gegenbild zur lärmenden Welt der Städte. Nikolaj Vasil'evič Gogol' (1809-1852) beschrieb die karge Wald- und Steppenlandschaften als romantische, russische Natur. Turgenev feierte die Ruhe in der russischen Landschaft. ${ }^{178}$ In den Werken beider Autoren erscheinen Gegenden abseits der Städte als erstrebenswertes Idyll. Dieses Narrativ war Teil eines literarischen und gesellschaftlichen Trends, der die russische Landbevölkerung und ihre Lebensform stark idealisierte und hoffte, hier eine nationale russische Identität zu finden. ${ }^{179} \mathrm{Um}$ die Wende zum 20. Jahrhundert lösten realistische Schilderungen die Idealvorstellungen der bäuerlichen Welt ab. Čechov und Ivan Alekseevič Bunin (18701953) thematisierten in ihren Texten, wie sich der gesellschaftliche Wandel der Industrialisierung auch auf die Dorfbevölkerung auswirkte: ${ }^{180}$ Auch diese heile Welt war an die neue Zeit verlorengegangen. Die gescheiterten Persönlichkeiten in Čechovs Dramen und Texten zeugen neben dem Verlust des Dorfes vom Verschwinden der gesamten alten, vertrauten Welt. Der Kirschgarten, Symbol der traditionellen aristokratischen Lebensweise im Zarenreich fällt dem Profitstreben eines Kaufmanns und ehemaligen Leibeigenen zum Opfer. Unsicherheit, Leere und das Gefühl, mit der neuen Zeit nicht Schritt halten zu können, keine positive Zukunft vor sich zu haben, prägen die schwermütige

dem Titel des degenerierten russischen Aristokraten hinterherzulaufen." Zitiert nach: Schlögel: Jenseits des Großen Oktober, S. 237.

177 Dowler: Russia in 1913, S. 246. Zu Gork'kij und dem Thema der Fabrik siehe auch: Gottfried Schramm: Von Puschkin bis Gorki. Dichterische Wahrnehmungen einer Gesellschaft im Wandel, Freiburg i. Br. 2008, S. 334-336.

${ }_{17} 8$ Klaus Gestwa: Primat der Umgestaltung und Pathos des Bewahrens. Literarische Landschafts- und Gesellschaftspanoramen im industriellen Wandel des zarischen Russlands und der frühen Sowjetunion, in: Zeitschrift für Geschichtswissenschaft, Jg. 51/2003, Heft 12, S. 1068-1097, S. 1081-1082.

179 Andrew Donskov: The Changing Image of the Peasant in Nineteenth-Century Drama, Helsinki 1972; Cathy A. Frierson: Peasant Icons. Representations of Rural People in Late 19th Century Russia, Oxford, New York 1993, S. 32-53. Das Bild des Landlebens unterschied sich stark von der Realität in den russischen Dörfern. Hierzu: Christine D. Worobec: Peasant Russia. Family and Community in the Post-Emancipation Period, Princeton 1991; Engel: Between the Fields and the City.

180 Thomas Marullo: Ivan Bunin's „Derevnja“. The Demythologization of the Peasant, in: Russian Language Journal, Jg. 31/1978, Heft 109, S. 79-100; Rolf-Dieter Kluge: Vom kritischen zum sozialistischen Realismus. Die literarische Tradition in Rußland 18801925, München 1973, S. 95-97; Donald Rayfield, Understanding Chekhov. A Critical Study of Chekhov's Prose and Drama, London 1999, S. 183-197. 
Grundstimmung des Werks. ${ }^{181}$ Gewinner der neuen Zeit gibt es bei Čechov keine. ${ }^{182}$

Viele Künstler nahmen die zunehmende Zersplitterung der Gesellschaft wahr. ${ }^{183}$ Die Symbolisten sahen ihre Aufgabe in der Suche nach einer neuen nationalen Einheit in der Kunst. Diese sollte, so die Wunschvorstellung, mit ihrer Ästhetik neue Menschen mit einer neuen Moral hervorbringen, die die Welt verändern würden. ${ }^{184}$ Großstädte und Technik beschrieben sie als vom Menschen geschaffene Ungetüme: Sie drohten alles zu verschlingen. Den Symbolisten erschienen die Zerstörung und das Verschwinden der Natur durch rational geplante und nach geometrischen Vorgaben gebaute Großstädte als elementare Bedrohung. 185

\subsection{Fazit}

Das Zarenreich entwickelte sich im Laufe des 19. Jahrhunderts und bis zum Beginn des Ersten Weltkriegs zur fünftgrößten Wirtschaftsmacht der Erde. Obwohl das Land absolut gesehen bis zur Oktoberrevolution ein Agrarstaat blieb, ${ }^{186}$ entstanden besonders in der zweiten Hälfte des 19. Jahrhunderts große industrielle Zentren mit Unternehmen, die mehrere tausend Arbeiter beschäftigten. Staatliche Maßnahmen sowie privatwirtschaftliches Engagement, gerade auch von ausländischen Unternehmern, ließen die russische Wirtschaft hohe Wachstumszahlen erreichen, so dass trotz der zahlreichen Krisen eine positive Bilanz gezogen werden kann.

Aufgrund seiner geringen wirtschaftlichen Bedeutung dominierten privatwirtschaftliche Initiativen das Gewerbe der Fotografie. Das neue visuelle Medium erreichte das Zarenreich in der ersten Phase der Industrialisierung 1839 und in den $1840 e r$ Jahren. Aus der neuen Technik entwickelte sich ein eigenständiges Gewerbe, das in den Metropolen sowie in kleinen Provinzstädten Menschen unterschiedlicher sozialer Herkunft ergriffen und Ateliers

181 Francis Fergusson: The Cherry Orchard. A Theater-Poem of the Suffering of Change, in: Robert L. Jackson (Hrsg.): Chekhov. A Collection of Critical Essays, Englewood Cliffs 1967, S. $147-160$.

182 Maria Deppermann: Anton Tschechow - Onkel Wanja, in: Bodo Zelinsky (Hrsg.): Das russische Drama, Düsseldorf 1986, S. 147-161, S. 149.

183 Steinberg: Petersburg, S. 34 .

184 Dowler: Russia in 1913, S. 256-263.

185 Sigrid Nolda: Symbolistischer Urbanismus. Zum Thema der Großstadt im russischen Symbolismus, Frankfurt a. M. 1980.

1861900 machte die Landwirtschaft noch immer 53 Prozent des Nationaleinkommens aus, währen die Industrie nur 21 Prozent beisteuerte. Haumann: Geschichte Russlands, S. 365. 
eröffneten. Die Kosten der Fotografien und der Aufwand für eine Aufnahme verringerten sich stetig, so dass die neuen Bilder einen festen Platz im russischen Alltagsleben eroberten. Mit ihren Kameras hielten die Fotografen die Auswirkungen der wirtschaftlichen Veränderungen fest und dokumentierten den Wandel der russischen Gesellschaft, den die Industrialisierung hervorrief.

Der Ausbau der Wirtschaft im Zarenreich war in der russischen Gesellschaft umstritten. Bis zum Ersten Weltkrieg dauerten innerhalb der Eliten die Auseinandersetzungen darüber an, welches Entwicklungsmodell das beste für die russische Wirtschaft sei. Die unterschiedlichen Interessensgruppen waren nicht homogen, sie verliefen quer durch politische Lager und Schichten. ${ }^{187}$ Die kritische Auseinandersetzung mit der technischen Moderne sowie die Ablehnung der gesellschaftlichen Entwicklung kann im Kontext einer kulturpessimistischen Grundstimmung gesehen werden, die um die Wende zum 20. Jahrhunderts auch in westeuropäischen Staaten verbreitet war. ${ }^{188}$ Die radikalen gesellschaftlichen Veränderungen im Zarenreich und die lange Tradition im Aushandeln des eigenen Verhältnisses gegenüber Westeuropa äußerten sich im Russischen Reich in einer besonders kritischen Haltung gegenüber der neuen Zeit. 189

Diese Diskussionen bildeten den Hintergrund, vor dem sich russische Unternehmer mit ihren Betrieben möglichst in positivem Licht zu positionieren suchten. Die russische Industrie entwickelte sich bis zur Jahrhundertwende

187 Während der ersten Hälfte des 19. Jahrhunderts dominierten allerdings Mitglieder des Adels die Debatten. David Saunders: Russia in the Age of Reaction and Reform. 1801-1881, London 1992, S. 163 .

188 Michel Grunewald; Uwe Puschner (Hrsg.): Krisenwahrnehmungen in Deutschland um 1900. Zeitschriften als Foren der Umbruchszeit im wilhelminischen Reich, Frankfurt a. M. 2010; Pier Paolo Portinaro: Kulturpessimismus und die Grenzen der Entzauberung. Diagnosen zur Technik, Kultur und Politik nach der Jahrhundertwende, in: Rüdiger vom Bruch; Friedrich Wilhelm Graf; Gangolf Hübner (Hrsg.): Kultur und Kulturwissenschaften um 1900. Krise der Moderne und Glaube an die Wissenschaft, Wiesbaden, Stuttgart 1989, S. 175-196; Glyn Turton: Turgenev and the Context of English Literature 1850-1900, London 1992, S. 147; Eugen Weber: France. Fin de siècle, Cambridge, Mass. 1986, S. 105-129; Monika Fludernik (Hrsg.): Fin de siècle, Trier 2002.

189 In Deutschland und Frankreich waren die Stimmen, die die Industrialisierung positiv sahen und die nationale Entwicklung der Industrie feierten ein weit verbreitetes Phänomen und Teil der nationalen Selbstinszenierung. Boch: Fabriken, S. 538-539; Monika Gibas: Repräsentationen von Industrielandschaften in raumbezogenen Identitätsdiskursen des 19. und 20. Jahrhunderts, in: Detlef Altenburg; Lothar Ehrlich; Jürgen John (Hrsg.): Im Herzen Europas. Nationale Identitäten und Erinnerungskulturen, Köln 2008, S. 251-275, S. 257-260; Jakob Vogel: Mythos Moderne. Die Technik in der nationalen Selbstdarstellung in Europa, in: Detlef Altenburg; Lothar Ehrlich; Jürgen John (Hrsg.): Im Herzen Europas. Nationale Identitäten und Erinnerungskulturen, Köln 2008, S. 105-120, S. 106-111. 
zu einem wichtigen Wirtschaftsfaktor, während sich die Fotografie gleichzeitig als attraktives Bildmedium einen festen Platz innerhalb der Gesellschaft des Zarenreichs eroberte. Zu Wechselwirkungen zwischen beiden Bereichen kam es im Russischen Reich erst im letzten Drittel des 19. Jahrhunderts. Obwohl es vor 1870 nur wenige Fotografien von Fabriken gab, bedeutet dies jedoch nicht, dass das Motiv in den Bildwelten des Zarenreichs vollkommen abwesend war. 